\title{
Mathematical Modelling Co-existence of Diabetes and COVID-19 : Deterministic and Stochastic Approach
}

\author{
S. Anusha ${ }^{1}$ - S. Athithan ${ }^{1}$
}

Received: date / Accepted: date

\begin{abstract}
Diabetes plays a vital role in the epidemiological cause of COVID-19 widely. So that the study of COVID-19 morbidity along with diabetes is to be elevated for the better solutions of public healthcare policies. Even though the COVID-19 infection along with diabetes was present in the human body, COVID-19 only having the potential to infect others and diabetes can remain with the particular human so that we call it a co-existence of two diseases. The infection point of view is considered only in the COVID-19 part. Diabetes will not be transmitted from one to other. Based on these ideas, we have developed a mathematical model which consists of five compartments corresponding to five population classes, namely, diabetes susceptible, diabetes patients, COVID-19 susceptible, COVID-19 infected, and COVID-19 recovered class. We have shown the non-negativity and invariant region of the system and discussed the existence of equilibrium points. Our system exhibits two equilibrium points. The stabilities of those equilibria of the model are studied. Also, by using the next-generation matrix method the basic reproduction number $\left(R_{0}\right)$ is calculated. Further, we perform the sensitivity analysis of $R_{0}$ to see the efficacy and influence of each of the parameters involved in $R_{0}$ and observed that reduction of transmission coefficient $\left(\alpha_{1}\right)$ from diabetes susceptible class to diabetes class is the most critical factor to control the co-morbidity. We have attempted to fit our model with the data given by the World Health Organization(WHO) [1] and it also suits well with the data. The deterministic model is extended into the stochastic model and by using numerical simulations our results of stochastic and deterministic models are compared. Our numerical findings were illustrating the fact that the chance of getting COVID-19 by diabetes patients is high if they have come into contact with COVID-
\end{abstract}

S. Anusha

E-mail: anushas@srmist.edu.in

$\triangle$ S. Athithan

E-mail: athithas@srmist.edu.in

1 Department of Mathematics, Faculty of Engineering and Technology, SRM Institute of Science and Technology, Kattankulathur, Tamil Nadu-603203, India. 
19 infected people. The results also illustrate the robustness of our model from the eco-epidemiological perspective. It is also obtained and highlighted that the burden of diabetes and COVID-19 co-existence and the role of the $\alpha_{1}$ in the severity of the disease.

Keywords Diabetes/Diabetics · COVID-19/Corona virus - Sensitivity analysis · Co-infection $\cdot$ Deterministic model $\cdot$ Stochastic model $\cdot$ Stability analysis

Mathematics Subject Classification (2020) 34D20 - 65C30 - 92B05 - 37N25 · $34 \mathrm{~A} 34 \cdot 92 \mathrm{C} 50 \cdot 34 \mathrm{~F} 05 \cdot 92 \mathrm{D} 25$

\section{Introduction}

According to World Health Organization(WHO), the novel coronavirus disease 2019 or simply COVID-19 is an infectious disease caused by SARS-CoV-2 i.e Severe Acute Respiratory Syndrome CoronaVirus-2 [2,3,4,5]. Coronavirus transmission can occur from infected individuals to susceptible individuals via direct communication (contaminated hands) or through droplets spread by coughing or sneezing from an infected person or airborne spread. The symptoms of COVID-19 include fatigue, dry cough, fever, diarrhea, chest pain, loss of smell and taste senses, shortness of breath, and breathing difficulties. COVID-19 has been declared as a public health emergency on January 30, 2020 [6] and pandemic on March 11 2020[7] by WHO, due to its exponential worldwide growth.

As of May 31, 2021, the total confirmed cases of COVID-19 have reached over $170,108,031$ with more than $3,659,455$ deaths reported[1]. The morbidity and mortality vary across countries of the world with the highest reported in the United States [8](30,058,797 confirmed cases with 588,596 deaths), India [9](28,047,534 confirmed cases with 329,100 deaths), Brazil [10](16,471,600 confirmed cases with 461,057 deaths), France [11](5,566,214 confirmed cases with 108,558 deaths), Russia [12] $(5,071,917$ confirmed cases with 121,501 deaths $)$ and Turkey [13] $(5,242,911$ confirmed cases with 47,405 deaths).

Mortality rates are higher in older people and patients with pre-existing medical conditions like diabetes, chronic lung disease, hypertension, malignancy, cardiovascular disease, and cerebrovascular diseases [14], [15]. Recently, epidemiological studies are focusing on the co-infection of COVID-19 and comorbidities, because comorbidities have been recognized as a risk factor for increased COVID-19 cases and higher fatality [16], [17]. Also, various studies have shown that diabetes is one of the major co-morbidity associated with severe illness and death from COVID-19 infection [18, 19,20].

Diabetes is a chronic condition that occurs when there is an imbalanced level of glucose in the blood [21]. According to the report of the International Diabetes Federation (IDF), [22] and WHO [23], 463 million adults are having diabetes; nearly 760 billion USD was spent on healthcare; more than 310 million diabetes patients are living in urban areas and about $11.3 \%$ of deaths were due to diabetes.

According to the Centers for Disease Control and Prevention (CDC), [24], the rate of fatality from COVID-19 is up to $50 \%$ higher in patients with diabetes than 
in the individual who does not have diabetes [25]. In [26], [27], it was reported that $20-30 \%$ of patients who died from COVID-19 had diabetes. A survey conducted in China showed that the higher prevalent comorbidity in COVID-19 patients was diabetes with $8.2 \%$ [28].

However, the above attestations demonstrate the fact that co-infection of diabetes and COVID-19 have a poor prognosis and increased death rate. So, analyzing how diabetes aggravates COVID-19 outcomes can give us better insight to restrain the disease and development of effective public health policies. At a recent time, several researchers tried to study the effects of co-infection of diabetes and COVID-19 through their respective fields.

For example, in [29] the author mentioned that COVID-19 patients with diabetes are susceptible to lower extremity ischemic and other complicated diseases. Rimesh Pal and Sanjay [30] studied an unholy interaction of COVID-19 and diabetes. They found that concurrent COVID-19 makes glucose control difficult to treat due to fluctuations in blood with diabetes patients.

Furthermore, in [31] authors have presented co-infection related worries and subsequent outcomes. Here authors have suggested that improving knowledge and precaution measures are necessary for people with diabetes during the COVID-19 pandemic. Interesting research for diabetes and COVID-19 co-infection disease is carried out in [32], where authors have exhibited the fact that metformin treatment might benefit the COVID-19 patients with diabetes.

On the other hand, in mathematical modeling, there is a lack of research focus on studies dealing with co-infection of diabetes and COVID-19. Although, many mathematical models have been derived to understand the patterns of each disease (COVID-19 [33, 34, 35, 36, 37] and diabetes [38,39, 40, 41, 42]), as of now only one study [43] has explored diabetes and COVID-19 co-infection. This paper [43] emphasizes that the negative impact of quarantine and several strategies to the lifting of the quarantine. In addition, the author worked on the optimal control techniques to provide an optimal treatment guideline for diabetes and COVID-19 co-infected patients.

Motivated by the aforementioned study, in this paper, we analyze the dynamics of the co-infection of diabetes and COVID-19. Our aim is twofold:

(i) We framed a deterministic model that describes diabetes and COVID-19 coinfection. We show the existence of equilibrium and discussed its stability.

(ii) Actually, stochastic models are more realistic as compared to deterministic models. Indeed, the stochastic models are more capable of capturing randomness in epidemic disease [44, 45, 46, 47, 48]. So our proposed model is extended to a stochastic model to see the effects of stochastic perturbations.

The structure of the rest of the paper is as follows. In section 2, we have constructed a mathematical model, which is based on some basic assumptions. The section 3 , covers the basic properties of our system. In section 4, we have discussed the existence of the equilibrium points and in subsection 4.3 the basic reproduction number $\left(R_{0}\right)$ is calculated. In section 5, the sensitivity analysis of $R_{0}$ is demonstrated which helps us to find out the key parameters. In section 6, the stability of equilibrium points is investigated. In section 7, we tried to fit the data from WHO. In section 8, we present our derived stochastic model extended from the determin- 
istic model. In section 9, numerical simulations are provided to verify the analytical findings. Finally, in section 10 , we conclude some of the key findings and ecological implications of our research.

\section{Model formulation}

In this section we have proposed a mathematical model for co-infection of diabetes and COVID-19 disease. To derive our model equations, we divide the total population $N(t)$ into five compartments namely Diabetes susceptible class $S_{d}(t)$, Diabetes class $D(t)$, COVID-19 Susceptible class $S_{c}(t)$, COVID-19 Infected class $I_{c}(t)$ and COVID-19 Recovered class $R_{c}(t)$.

For developing the mathematical model, we make the following assumptions.

i. All compartments are mutually exclusive.

ii. Total population is given by $N(t)=S_{d}(t)+D(t)+S_{c}(t)+I_{c}(t)+R_{c}(t)$.

iii. The population of our model is homogeneously mixed. This means that each individual has an equal chance to enter into COVID-19 infected compartment.

iv. A simple mass-action type interaction for the disease transmission is considered.

v. Diabetes is a non-communicable disease caused by an imbalanced level of glucose. So there is no transmission of diabetes between two people.

vi. If either the production rate of insulin in the pancreas is low or the body cannot use the produced insulin effectively, then diabetes susceptible individual $\left(S_{d}\right)$ moves to diabetes class $(D)$ at a rate of $\alpha_{1}$.

vii. The COVID-19 susceptible population $\left(S_{c}\right)$ is composed of individuals who have not yet been infected by the COVID-19 but may be infected through contact with COVID-19 infected people.

viii. Since COVID-19 is not age-specific i.e. any adult age group can be affected if they come into contact with infected people so that we considered the transmission between

$\star S_{d}$ to $I_{c}$ at the rate of $\alpha_{2}$

$\star S_{c}$ to $I_{c}$ at the rate of $\beta$

* $D$ to $I_{c}$ at the rate of $\rho$

ix. $\gamma$ is the rate at which infective individuals in $I_{c}$ recovered from the disease enter to the recovered class $R_{c}$.

$\mathrm{x}$. The individuals are losing their immunity against COVID-19 and hence, moving to COVID-19 susceptible class.

xi. The general people may stay in the recovered class itself but the diabetes patients were automatically moved to their native diabetes class. 
Table 1: Descriptions of parameters involved in the system (1).

\begin{tabular}{cl}
\hline Parameter & Description \\
\hline$\Lambda$ & Rate of recruitment to the susceptible COVID-19 class $S_{c}$ \\
$\Phi$ & Rate of recruitment to the susceptible Diabetes class $S_{d}$ \\
$\beta$ & Rate of progression from $S_{c}$ to $I_{c}$ due to the transmission of COVID-19 virus \\
$\gamma$ & Rate of recovery of an individual due to treatment (may be with temporary immunity) \\
$\delta$ & Rate of movement of individuals from $R_{c}$ to $D$ \\
$\alpha_{1}$ & Rate of progression of individuals from $S_{d}$ to $D$ \\
$\alpha_{2}$ & Rate of disease transmission from $S_{d}$ to $I_{c}$ \\
$\rho$ & Rate of disease transmission from $D$ to $I_{c}$ \\
$\ell$ & Rate of movement of individuals from $R_{c}$ to $S_{c}$ due to loss of immunity \\
$\mu$ & Natural mortality rate \\
$\mu_{1}$ & Mortality rate due to COVID-19
\end{tabular}

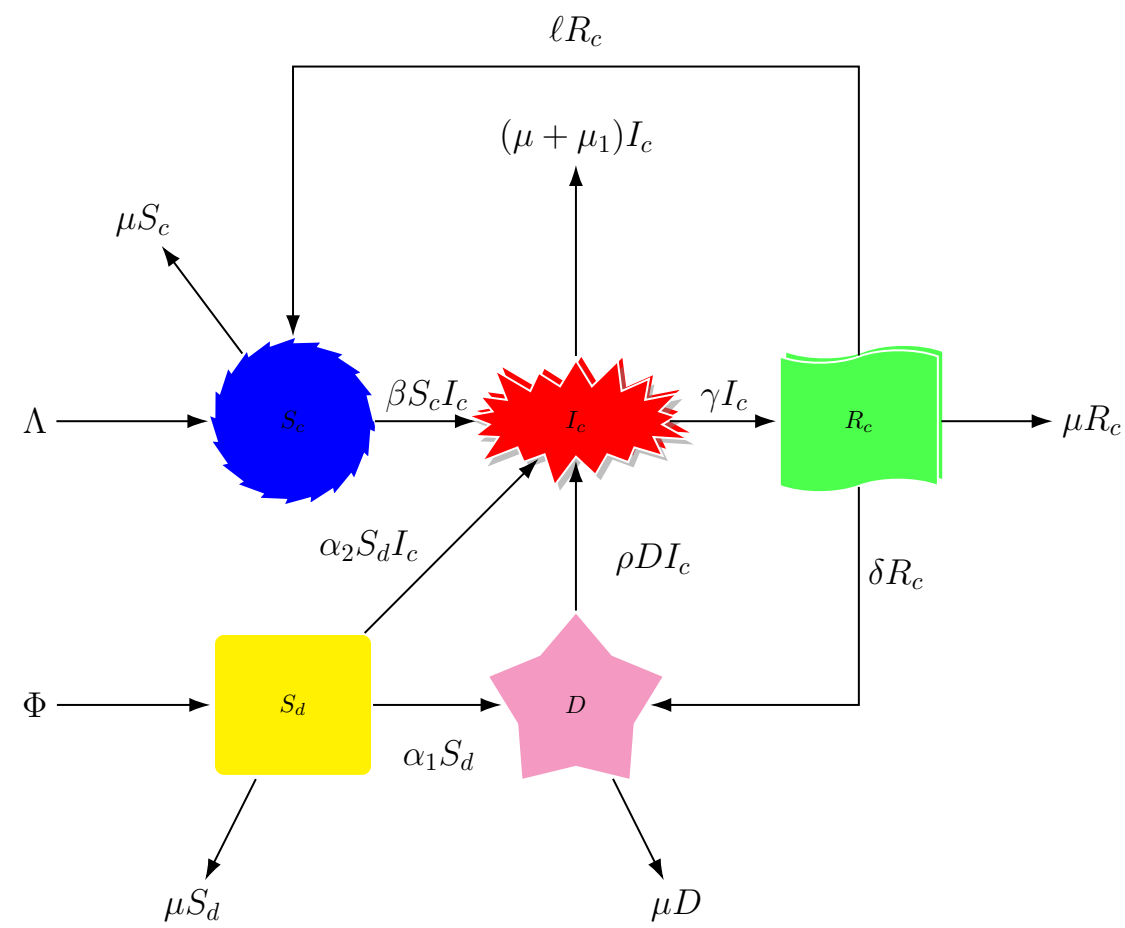

Fig. 1: Schematic diagram of the model (1) 
Based on these assumptions, the following mathematical model of co-infection of diabetes and COVID-19 has been formulated:

$$
\begin{aligned}
\frac{d S_{d}}{d t} & =\Phi-\alpha_{1} S_{d}-\alpha_{2} S_{d} I_{c}-\mu S_{d} \\
\frac{d D}{d t} & =\alpha_{1} S_{d}+\delta R_{c}-\rho D I_{c}-\mu D \\
\frac{d S_{c}}{d t} & =\Lambda+\ell R_{c}-\beta S_{c} I_{c}-\mu S_{c} \\
\frac{d I_{c}}{d t} & =\beta S_{c} I_{c}+\rho D I_{c}+\alpha_{2} S_{d} I_{c}-\gamma I_{c}-\mu I_{c}-\mu_{1} I_{c} \\
\frac{d R_{c}}{d t} & =\gamma I_{c}-\ell R_{c}-\delta R_{c}-\mu R_{c}
\end{aligned}
$$

with initial conditions

$$
S_{d}(0)>0, \quad D(0) \geq 0, \quad S_{c}(0)>0, \quad I_{c}(0) \geq 0, \quad R_{c}(0) \geq 0 .
$$

Fig. 1 1 represents the flow of individuals from one class to the other. Also, the biological meanings of parameters involved in system (1) are given in Table 1 .

\section{Qualitative properties of the model}

3.1 Non-negativity of solutions

Theorem 1 Every solution of system (1) with initial conditions (2) exists in the interval $[0, \infty)$ and $S_{d}(t)>0, D(t) \geq 0, S_{c}(t)>0, I_{c}(t) \geq 0, R_{c}(t) \geq 0$ for all $t \geq 0$.

Proof Since the right hand side of system (1) is completely continuous and locally Lipschitzian on $C$ (space of continuous functions), the solution $\left(S_{d}(t), D(t), S_{c}(t), I_{c}(t), R_{c}(t)\right)$ of (11) with initial conditions (2) exists and is unique on $[0, \xi)$, where $0<\xi \leq+\infty$.

From the fourth equation of (1), we have

$$
I_{c}(t)=I_{c}(0) \exp \left[\int_{0}^{t}\left\{\beta S_{c}(s)+\rho D(s)+\alpha_{2} S_{d}(s)-\left(\gamma+\mu+\mu_{1}\right)\right\} d s\right] \geq 0
$$

Also, from the fifth equation of (1), we get

$$
\begin{aligned}
& \frac{d R_{c}}{d t} \geq-[\ell+\delta+\mu] R_{c}(t) \quad\left[\because I_{c}(t) \geq 0\right] \\
& \Longrightarrow R_{c}(t) \geq R_{c}(0) \exp [-(\ell+\delta+\mu) t] \geq 0
\end{aligned}
$$

It follows from the third equation of the system (1) that,

$$
\frac{d S_{c}}{d t} \geq \Lambda-\left[\beta I_{c}(t)+\mu\right] S_{c}(t) \quad\left[\because R_{c}(t) \geq 0\right]
$$


Co-existence of Diabetes and COVID-19

7

we thus have

$$
\frac{d}{d t}\left[S_{c}(t) \exp \left\{\mu t+\int_{0}^{t} \beta I_{c}(s) d s\right\}\right] \geq \Lambda \exp \left\{\mu t+\int_{0}^{t} \beta I_{c}(s) d s\right\}
$$

Hence,

$$
S_{c}(t) \exp \left\{\mu t+\int_{0}^{t} \beta I_{c}(s) d s\right\}-S_{c}(0) \geq \int_{0}^{t} \Lambda \exp \left\{\mu t+\int_{0}^{t} \beta I_{c}(s) d s\right\} d t
$$

so that

$$
\begin{array}{r}
S_{c}(t) \geq S_{c}(0) \exp \left[-\left\{\mu t+\int_{0}^{t} \beta I_{c}(s) d s\right\}\right]+\exp \left[-\left\{\mu t+\int_{0}^{t} \beta I_{c}(s) d s\right\}\right] \\
\times\left[\int_{0}^{t} \Lambda \exp \left\{\mu t+\int_{0}^{t} \beta I_{c}(s) d s\right\} d t\right]>0
\end{array}
$$

Similarly, it follows from the first equation of the system (1) that,

$$
\frac{d S_{d}}{d t}=\Phi-\left[\left(\alpha_{1}+\mu\right)+\alpha_{2} I_{c}\right] S_{d}
$$

we thus have

$$
\frac{d}{d t}\left[S_{d}(t) \exp \left\{\left(\alpha_{1}+\mu\right) t+\int_{0}^{t} \alpha_{2} I_{c}(s) d s\right\}\right]=\Phi \exp \left\{\left(\alpha_{1}+\mu\right) t+\int_{0}^{t} \alpha_{2} I_{c}(s) d s\right\}
$$

Hence

$S_{d}(t) \exp \left\{\left(\alpha_{1}+\mu\right) t+\int_{0}^{t} \alpha_{2} I_{c}(s) d s\right\}-S_{d}(0)=\int_{0}^{t} \Phi \exp \left\{\left(\alpha_{1}+\mu\right) t+\int_{0}^{t} \alpha_{2} I_{c}(s) d s\right\} d t$

so that

$$
\begin{array}{r}
S_{d}(t)=S_{d}(0) \exp \left[-\left\{\left(\alpha_{1}+\mu\right) t+\int_{0}^{t} \alpha_{2} I_{c}(s) d s\right\}\right]+\exp \left[-\left\{\left(\alpha_{1}+\mu\right) t+\int_{0}^{t} \alpha_{2} I_{c}(s) d s\right\}\right] \\
\times\left[\int_{0}^{t} \Phi \exp \left\{\left(\alpha_{1}+\mu\right) t+\int_{0}^{t} \alpha_{2} I_{c}(s) d s\right\} d t\right]>0
\end{array}
$$

Finally, from the second equation of (1), we have

$$
\begin{array}{cc} 
& \frac{d D}{d t} \geq-\left[\rho I_{c}(t)+\mu\right] D(t) \quad\left[\because S_{d}(t)>0\right] \\
\Longrightarrow & D(t) \geq D(0) \exp \left\{-\left(\mu t+\int_{0}^{t} \rho I_{c}(s) d s\right)\right\} \geq 0
\end{array}
$$

Therefore, we can see that

$$
S_{d}(t)>0, D(t) \geq 0, S_{c}(t)>0, I_{c}(t) \geq 0, R_{c}(t) \geq 0 \quad \forall t \geq 0
$$

This completes the proof. 
3.2 Invariant region

Theorem 2 The feasible region $\Omega$ defined by

$$
\Omega=\left\{\left(S_{d}(t), D(t), S_{c}(t), I_{c}(t), R_{c}(t)\right) \in \mathbb{R}_{+}^{5}: 0<N \leq \frac{\chi}{\mu}\right\}
$$

with initial conditions $S_{d}(0)>0, D(0) \geq 0, S_{c}(0)>0, I_{c}(0) \geq 0, R_{c}(0) \geq 0$ are positively invariant.

Proof Adding the equations of the system (1) we obtain

$$
\frac{d N}{d t}=\chi-\mu N-\mu_{1} I_{c} \leq \chi-\mu N \quad\left[\because I_{c} \geq 0\right]
$$

The solution $N(t)$ of the differential equation (3) has the following property,

$$
\Longrightarrow 0<N(t) \leq N(0) e^{-\mu t}+\frac{\chi}{\mu}\left(1-e^{-\mu t}\right)
$$

where $\chi=\Lambda+\Phi$ and $N(0)$ represents the sum of the initial values of the variables. As $t \rightarrow \infty, 0<N \leq \frac{\chi}{\mu}$. So, if $N(0) \leq \frac{\chi}{\mu}$, then $\lim _{t \rightarrow \infty} N(t) \leq \frac{\chi}{\mu}$. This means that $\frac{\chi}{\mu}$ is the upper bound of $N$. On the other hand, if $N(0)>\frac{\chi}{\mu}$, then $N(t)$ will decrease to $\frac{\chi}{\mu}$. This means that if $N(0)>\frac{\chi}{\mu}$, then the solution $\left(S_{d}(t), D(t), S_{c}(t), I_{c}(t), R_{c}(t)\right)$ enters $\Omega$ or approach it asymptotically. Hence it is positively invariant under the flow induced by the system (1). Thus in $\Omega$, the model (1) is well-posed epidemiologically and mathematically. Hence it is sufficient to study the dynamics of the model in $\Omega$.

\section{Existence of equilibrium}

In this section, we analyze the existence of equilibrium points of the system (1). System (1) has two types of equilibrium points:

1. Diseases Free Equilibrium (DFE) :- $E^{(0)}=\left(S_{d}^{0}, 0, S_{c}^{0}, 0,0\right)$

2. Endemic Equilibrium (EE) :- $E^{(1)}=\left(S_{d}^{*}, D^{*}, S_{c}^{*}, I_{c}^{*}, R_{c}^{*}\right)$

4.1 Diseases Free Equilibrium

In Diseases Free Equilibrium (DFE) $E^{(0)}=\left(S_{d}^{0}, 0, S_{c}^{0}, 0,0\right)$, disease is absent.

$$
\begin{gathered}
S_{d}^{0}=\frac{\Phi}{\alpha_{1}+\mu} \\
S_{c}^{0}=\frac{\Lambda}{\mu}
\end{gathered}
$$


4.2 Endemic Equilibrium

At an Endemic Equilibrium (EE) $E^{(1)}=\left(S_{d}^{*}, D^{*}, S_{c}^{*}, I_{c}^{*}, R_{c}^{*}\right)$, disease is present and the followings hold:

$$
\begin{gathered}
S_{d}>0, \quad D>0, \quad S_{c}>0, \quad I_{c}>0, \quad R_{c}>0 \\
\frac{d S_{d}}{d t}=\frac{d D}{d t}=\frac{d S_{c}}{d t}=\frac{d I_{c}}{d t}=\frac{d R_{c}}{d t}=0
\end{gathered}
$$

Solving the equations of system (1) at the equilibrium state we get

$$
\begin{aligned}
R_{c}^{*} & =h_{1} I_{c}^{*} \\
S_{c}^{*} & =\frac{\Lambda+\ell h_{1} I_{c}^{*}}{\beta I_{c}^{*}+\mu} \\
S_{d}^{*} & =\frac{\Phi}{\alpha_{2} I_{c}^{*}+k_{1}} \\
D^{*} & =\frac{\alpha_{1} \Phi+\alpha_{2} \delta h_{1}\left(I_{c}^{*}\right)^{2}+\delta h_{1} k_{1} I_{c}^{*}}{\left(\alpha_{2} I_{c}^{*}+k_{1}\right)\left(\rho I_{c}^{*}+\mu\right)}
\end{aligned}
$$

where

$$
k_{1}=\alpha_{1}+\mu, \quad k_{2}=\gamma+\mu+\mu_{1}, \quad k_{3}=\ell+\delta+\mu, \quad h_{1}=\frac{\gamma}{k_{3}}
$$

Now, putting the values of $S_{d}^{*}, D^{*}, S_{c}^{*}, R_{c}^{*}$ into the fourth equation of (1) and simplifying we obtain

$$
A_{1}\left(I_{c}^{*}\right)^{3}+A_{2}\left(I_{c}^{*}\right)^{2}+A_{3}\left(I_{c}^{*}\right)+A_{4}=0
$$

where

$$
\begin{aligned}
A_{1}= & -\frac{\beta \alpha_{2} \rho}{k_{3}}\left(\gamma \mu+\mu k_{3}+\mu_{1} k_{3}\right)<0 \\
A_{2}= & \beta \ell h_{1} \alpha_{2} \mu+\beta \Lambda \alpha_{2} \rho+k_{1} \rho \beta \ell h_{1}+\rho h_{1} k_{1} \beta \delta+\rho \alpha_{2} \delta h_{1} \mu+\alpha_{2} \Phi \beta \rho \\
& -k_{2} \alpha_{2} \mu \beta-k_{2} \rho k_{1} \beta-k_{2} \alpha_{2} \rho \mu \\
A_{3}= & \beta \Lambda \alpha_{2} \mu+\beta \Lambda k_{1} \rho+\beta \ell h_{1} k_{1} \mu+\rho \alpha_{1} \Phi \beta+\rho h_{1} k_{1} \delta \mu+\alpha_{2} \Phi \mu \rho+ \\
& \alpha_{2} \Phi \beta \mu-k_{2} \alpha_{2} \mu^{2}-k_{2} \rho k_{1} \mu-k_{2} k_{1} \beta \mu \\
A_{4}= & \beta \Lambda \mu k_{1}+\rho \alpha_{1} \Phi \mu+\alpha_{2} \Phi \mu^{2}-k_{1} k_{2} \mu^{2}
\end{aligned}
$$

Obviously $A_{1}$ is negative, However, the signs of $A_{2}, A_{3}, A_{4}$ are not obvious. Now, applying Descartes's rule of signs in equation (4) we obtain some of the results. These results are summarized in the Table 2 .

From Table 2, it is easy to observe that if $A_{4}$ is positive, then there exists at least one positive value for $I_{c}^{*}$. i.e, at least one non-trivial endemic equilibrium. Summarizing the above discussions we arrive to the following result:

Theorem 3 If $A_{4}$ in equation (4) is positive, then there exists at least one non-trivial endemic equilibrium of the system (1). 
Table 2: Existence of positive roots.

\begin{tabular}{cllllll}
\hline Cases & $A_{1}$ & $A_{2}$ & $A_{3}$ & $A_{4}$ & No. of sign changes & No. of possible positive root \\
\hline 1 & - & - & - & - & 0 & No root \\
2 & - & + & + & - & 2 & Two root \\
3 & - & + & - & - & 2 & Two root \\
4 & - & - & + & - & 2 & Two root \\
5 & - & + & + & + & 1 & Unique \\
6 & - & - & + & + & 1 & Unique \\
7 & - & - & - & + & 1 & Unique \\
8 & - & + & - & + & 3 & Atmost 3 possible roots \\
\hline
\end{tabular}

\subsection{The basic reproduction number}

The basic reproduction number $\left(R_{0}\right)$ for this proposed model (1) is defined as the average number of new infective individuals generated by a single infective individual during his/her infected period when introduced into a susceptible population.

One of the theoretical methods for the estimation of the $R_{0}$ is by the construction of the next-generation matrix for the system (1) as follows [49], [50]:

$$
\begin{gathered}
\mathcal{F}=\left(\begin{array}{c}
\alpha_{1} S_{d}+\delta R_{c} \\
\beta S_{c} I_{c}+\rho D I_{c}+\alpha_{2} S_{d} I_{c} \\
\gamma I_{c}
\end{array}\right) \quad \mathcal{V}=\left(\begin{array}{c}
\rho D I_{c}+\mu D \\
\gamma I_{c}+\mu I_{c}+\mu_{1} I_{c} \\
\ell R_{c}+\delta R_{c}+\mu R_{c}
\end{array}\right) \\
F=\text { Jacobian of } \mathcal{F} \text { at } E^{(0)}=\left(\begin{array}{ccc}
0 & 0 & \delta \\
0 & \beta S_{c}^{0}+\alpha_{2} S_{d}^{0} & 0 \\
0 & \gamma & 0
\end{array}\right) \\
V=\text { Jacobian of } \mathcal{V} \text { at } E^{(0)}=\left(\begin{array}{ccc}
\mu & 0 & 0 \\
0 & \gamma+\mu+\mu_{1} & \\
0 & 0 & \ell+\delta+\mu
\end{array}\right)
\end{gathered}
$$

It follows that

$$
F V^{-1}=\left(\begin{array}{ccc}
0 & 0 & \frac{\delta}{\ell+\delta+\mu} \\
0 \frac{\beta S_{c}^{0}+\alpha_{2} S_{d}^{0}}{\gamma+\mu+\mu_{1}} & 0 \\
0 \frac{\gamma}{\gamma+\mu+\mu_{1}} & 0
\end{array}\right)
$$

The basic reproduction number $\left(R_{0}\right)$ of model $(1)$ is given by the largest eigenvalue of $F V^{-1}$, and is obtained as,

$$
R_{0}=\frac{\beta \Lambda\left(\alpha_{1}+\mu\right)+\alpha_{2} \Phi \mu}{\mu\left(\alpha_{1}+\mu\right)\left(\gamma+\mu+\mu_{1}\right)}
$$




\section{Sensitivity Analysis}

The basic reproduction number $\left(R_{0}\right)$ of system (1) depends on eight parameters, namely, recruitment rate of COVID-19 and diabetes susceptible ( $\Lambda$ and $\Phi$ respectively), disease progression rate from $S_{c}$ to $I_{c}$ and $R_{c}$ to $D$ ( $\beta$ and $\delta$ respectively), transmission rate by an infective from $S_{c}$ to $I_{c}\left(\alpha_{1}\right)$, rate of movement from $S_{d}$ to $D$ due to imbalanced glucose level $\left(\alpha_{2}\right)$, recovery rate of COVID-19 individuals with temporary immunity $(\gamma)$, natural mortality rate $(\mu)$ and mortality rate due to COVID$19\left(\mu_{1}\right)$. Among those parameters, we can not control some of the parameters like $\mu$ and $\mu_{1}$. The partial derivatives shown below illustrate the sensitivity of the $R_{0}$ to changes in these parameters:

$$
\begin{aligned}
\frac{\partial R_{0}}{\partial \Lambda} & =\frac{\beta}{\mu\left(\gamma+\mu+\mu_{1}\right)}>0, \\
\frac{\partial R_{0}}{\partial \Phi} & =\frac{\alpha_{2}}{\left(\alpha_{1}+\mu\right)\left(\gamma+\mu+\mu_{1}\right)}>0, \\
\frac{\partial R_{0}}{\partial \beta} & =\frac{\Lambda}{\mu\left(\gamma+\mu+\mu_{1}\right)}>0, \\
\frac{\partial R_{0}}{\partial \gamma} & =-\frac{\beta \Lambda\left(\alpha_{1}+\mu\right)+\alpha_{2} \Phi \mu}{\mu\left(\alpha_{1}+\mu\right)\left(\gamma+\mu+\mu_{1}\right)^{2}}<0, \\
\frac{\partial R_{0}}{\partial \alpha_{1}} & =-\frac{\alpha_{2} \Phi}{\left(\alpha_{1}+\mu\right)^{2}\left(\gamma+\mu+\mu_{1}\right)}<0, \\
\frac{\partial R_{0}}{\partial \alpha_{2}} & =\frac{\Phi}{\left(\alpha_{1}+\mu\right)\left(\gamma+\mu+\mu_{1}\right)}>0
\end{aligned}
$$

Therefore, to study the sensitivity of $R_{0}$ of the parameters $\Lambda, \Phi, \beta, \gamma, \alpha_{1}$ and $\alpha_{2}$, following Arriola and Hyman [51], the normalized forward sensitivity index [52] with respect to each of those parameters are calculated as follows:

$$
\begin{aligned}
\Theta_{\Lambda} & =\frac{\Lambda}{R_{0}} \cdot \frac{\partial R_{0}}{\partial \Lambda}=\frac{\beta \Lambda\left(\alpha_{1}+\mu\right)}{\beta \Lambda\left(\alpha_{1}+\mu\right)+\alpha_{2} \Phi \mu} \\
\Theta_{\Phi} & =\frac{\Phi}{R_{0}} \cdot \frac{\partial R_{0}}{\partial \Phi}=\frac{\alpha_{2} \Phi \mu}{\beta \Lambda\left(\alpha_{1}+\mu\right)+\alpha_{2} \Phi \mu} \\
\Theta_{\beta} & =\frac{\beta}{R_{0}} \cdot \frac{\partial R_{0}}{\partial \beta}=\frac{\beta \Lambda\left(\alpha_{1}+\mu\right)}{\beta \Lambda\left(\alpha_{1}+\mu\right)+\alpha_{2} \Phi \mu} \\
\Theta_{\gamma} & =\frac{\gamma}{R_{0}} \cdot \frac{\partial R_{0}}{\partial \gamma}=-\frac{\gamma}{\left(\gamma+\mu+\mu_{1}\right)} \\
\Theta_{\alpha_{1}} & =\frac{\alpha_{1}}{R_{0}} \cdot \frac{\partial R_{0}}{\partial \alpha_{1}}=-\frac{\alpha_{1} \alpha_{2} \Phi \mu}{\beta \Lambda\left(\alpha_{1}+\mu\right)^{2}+\alpha_{2} \Phi \mu\left(\alpha_{1}+\mu\right)} \\
\Theta_{\alpha_{2}} & =\frac{\alpha_{2}}{R_{0}} \cdot \frac{\partial R_{0}}{\partial \alpha_{2}}=\frac{\alpha_{2} \Phi \mu}{\beta \Lambda\left(\alpha_{1}+\mu\right)+\alpha_{2} \Phi \mu}
\end{aligned}
$$



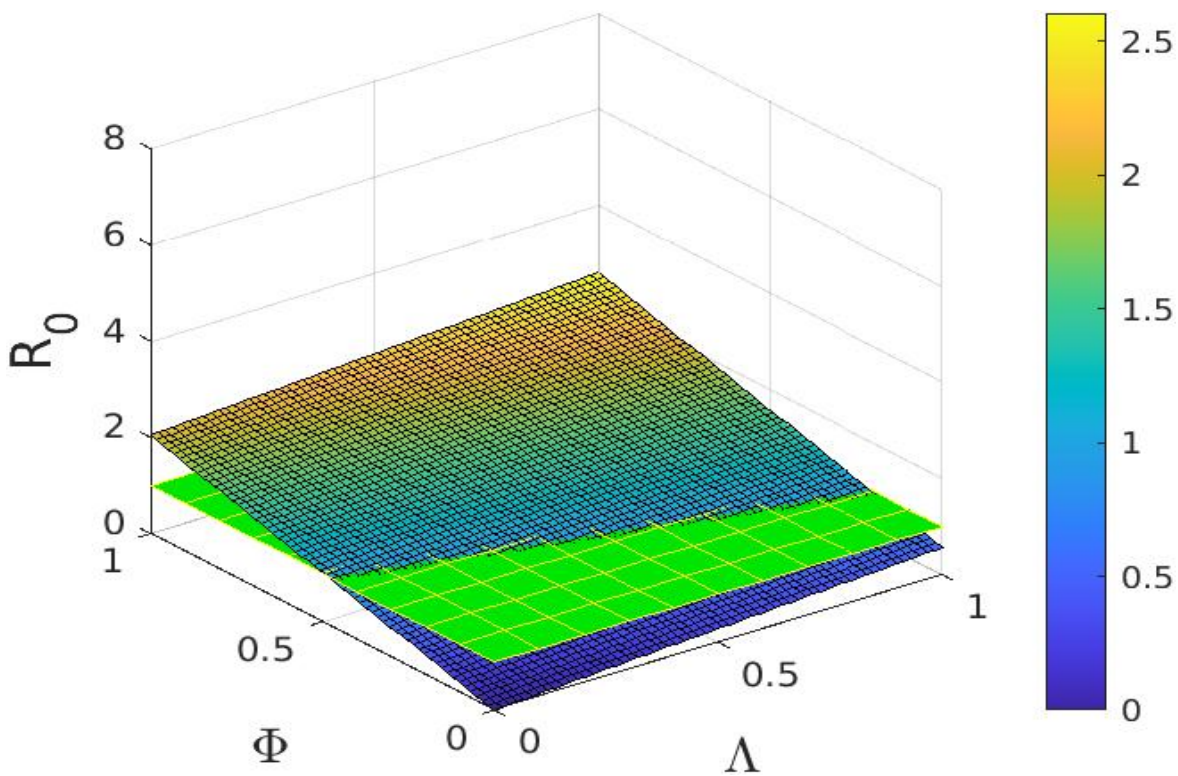

Fig. 2: Plot for variation in $R_{0}$ with respect to $\Lambda$ and $\Phi$

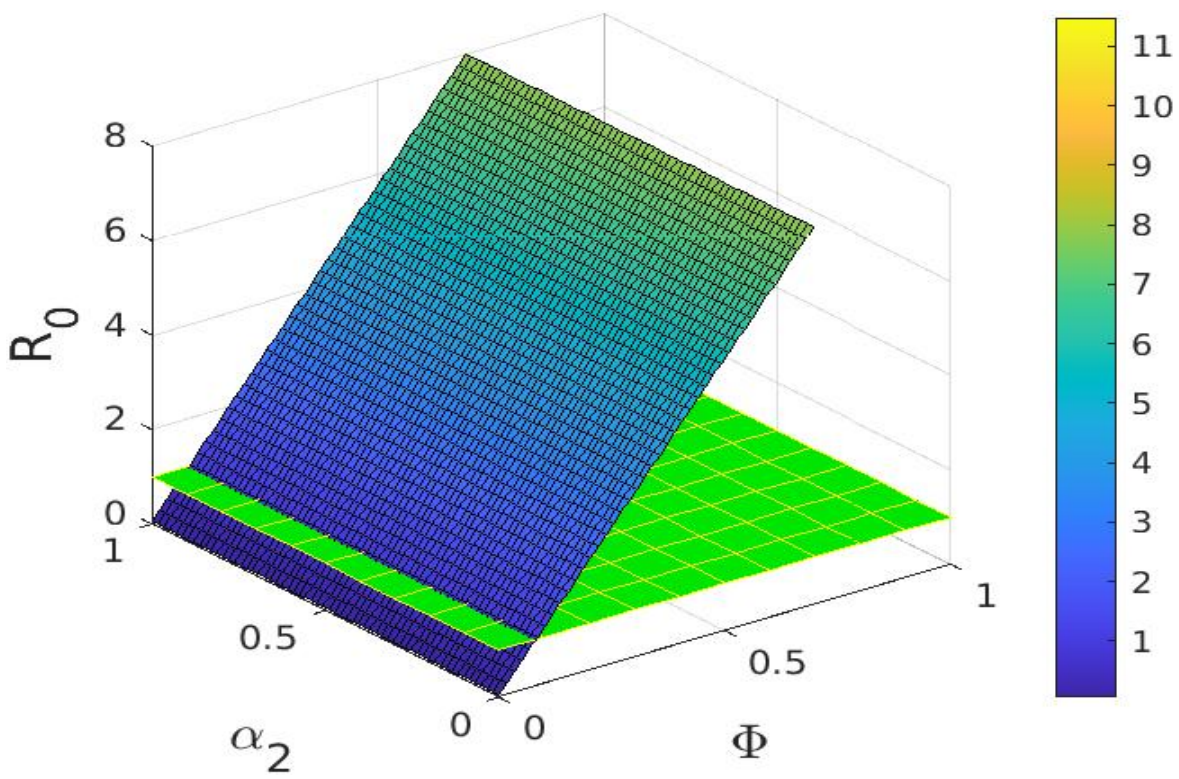

Fig. 3: Plot for variation in $R_{0}$ with respect to $\Phi$ and $\alpha_{2}$ 

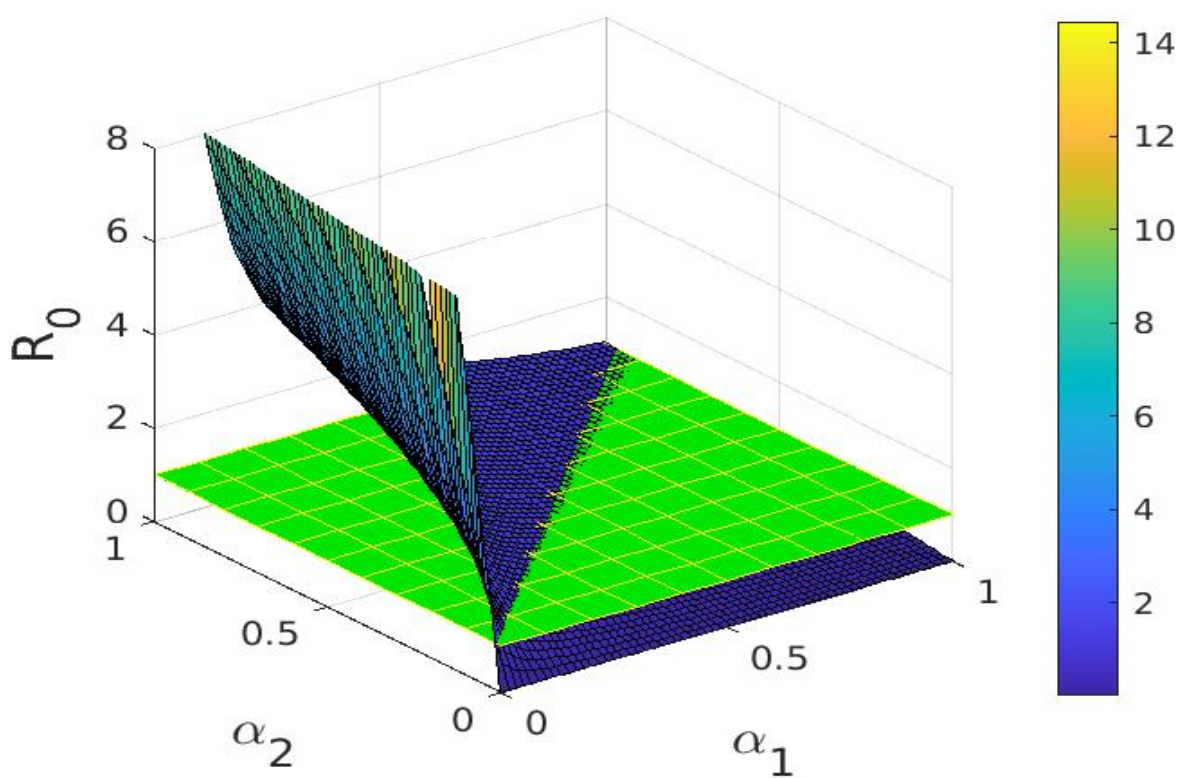

Fig. 4: Plot for variation in $R_{0}$ with respect to $\alpha_{1}$ and $\alpha_{2}$

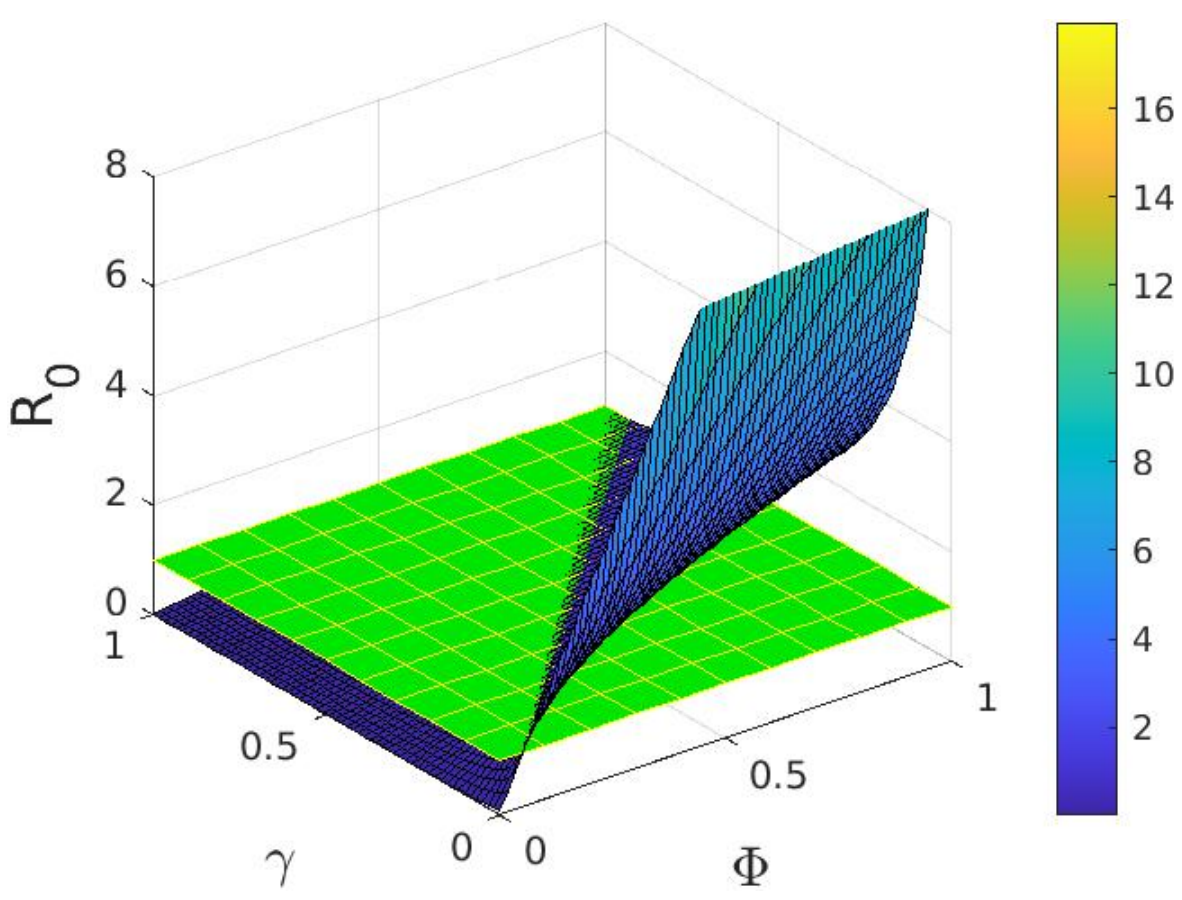

Fig. 5: Plot for variation in $R_{0}$ with respect to $\Phi$ and $\gamma$ 


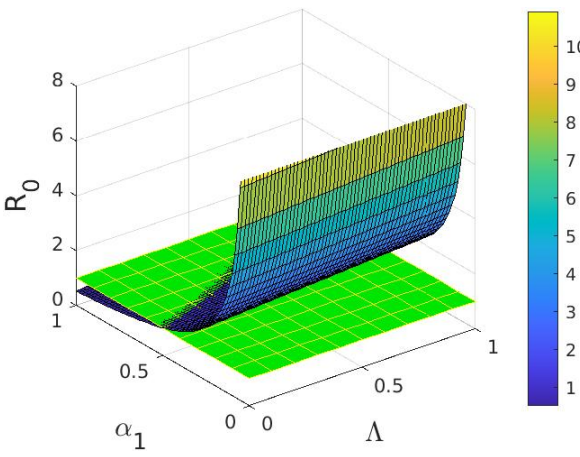

(a)

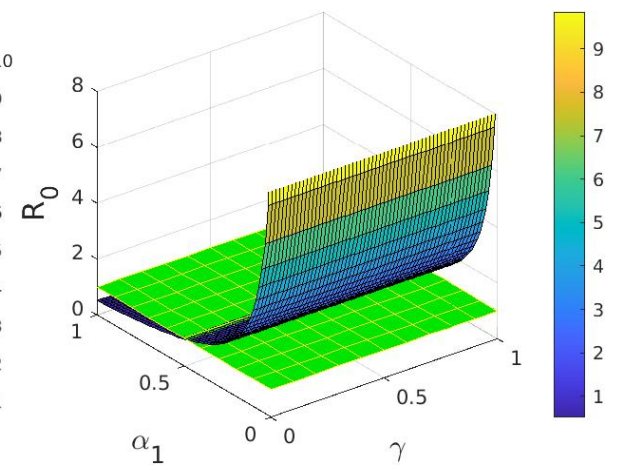

(b)

Fig. 6: Plot for variation in $R_{0}$ with respect to (a) $\Lambda$ and $\alpha_{1}$ and (b) $\gamma$ and $\alpha_{1}$

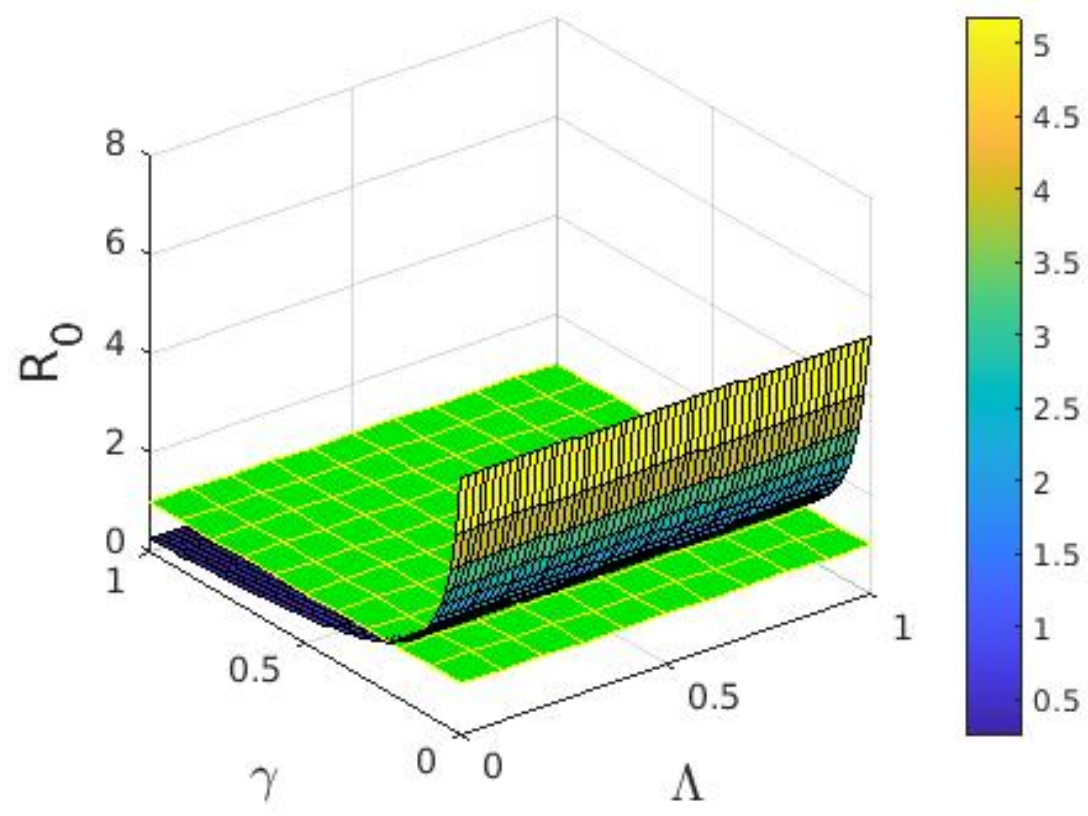

Fig. 7: Plot for variation in $R_{0}$ with respect to $\Lambda$ and $\gamma$

From the above expressions, it is clear that $\Theta_{\Lambda}, \Theta_{\Phi}, \Theta_{\beta}$ and $\Theta_{\alpha_{2}}$ are positive and $\Theta_{\gamma}, \Theta_{\alpha_{1}}$ are negative. This implies an increase in the parameters $\Lambda, \Phi, \beta$, and $\alpha_{2}$ leads to an increase in the value of the basic reproduction number, whereas the increase in the parameters $\gamma$ and $\alpha_{1}$ causes the decrease in basic reproduction number. As we see that $\Theta_{\Phi}=\Theta_{\alpha_{2}}$ which implies small changes in any of these parameters in $\Phi$ and 
$\alpha_{2}$ will have the same impact on reproduction number $R_{0}$. Similarly, $\Theta_{\Lambda}=\Theta_{\beta}$, we conclude that $\Lambda$ and $\beta$ have equal impact on basic reproduction number.

Here, Fig. 2-7, are demonstrating the impact of the parameters on reproduction number $R_{0} . \Lambda$ and $\Phi$ have an directly proportional relationship with $R_{0}$, i.e, the increase in any of them will cause an increase in $R_{0}$. This fact is demonstrated in Fig. 2. On the other hand, the parameter $\Phi$ which is the recruitment rate of diabetes has more effect on the basic reproduction number $R_{0}$ as compared to the parameter $\alpha_{2}$ which is shown in Fig. 3

Likewise, in Fig. 4 shows $\alpha_{2}$ has higher impact than $\alpha_{1}$ on $R_{0}$. In Fig. 5 we see the relation between $R_{0}, \Phi$ and $\gamma$. As expected increase in recruitment rate $\Lambda$ causes increase in basic reproduction number.

However, $\alpha_{1}$ have an inversely proportional relationship with basic reproduction number, i.e, increase of $\alpha_{1}$ will lead to a decrease in $R_{0}$ and if $\alpha_{1}$ decreases $R_{0}$ will increase which is reflected in Fig. 6 Next, in Fig. 7 the value of $R_{0}$ decreases with the increment of $\gamma$. As a result of our findings, we conclude that recovery $(\gamma)$ helps to eradicate the disease by reducing the $R_{0}$.

\section{Stability Analysis}

The stability results of different equilibria are summarized in the following theorems.

Theorem 4 The disease-free equilibrium $E^{(0)}=\left(S_{d}^{0}, 0, S_{c}^{0}, 0,0\right)$ of the system (1) is locally asymptotically stable if $R_{0}<1$ and unstable if $R_{0}>1$

Proof The variational matrix evaluated at the equilibrium point $E^{(0)}$ is given by

$$
M^{0}=\left(\begin{array}{ccccc}
-\alpha_{1}-\mu & 0 & 0 & -\alpha_{2} S_{d}^{0} & 0 \\
\alpha_{1} & -\mu & 0 & 0 & \delta \\
0 & 0 & -\mu & -\beta S_{c}^{0} & \ell \\
0 & 0 & 0 & b_{44} & 0 \\
0 & 0 & 0 & \gamma & -\ell-\delta-\mu
\end{array}\right)
$$

where $b_{44}=\beta S_{c}^{0}+\alpha_{2} S_{d}^{0}-\gamma-\mu-\mu_{1}=\left(\gamma+\mu+\mu_{1}\right)\left(R_{0}-1\right)$. Therefore, eigenvalues of the above matrix are

$\lambda_{1}=-\alpha_{1}-\mu, \quad \lambda_{2}=-\mu, \quad \lambda_{3}=-\mu, \lambda_{4}=\gamma+\mu+\mu_{1}\left(R_{0}-1\right), \quad \lambda_{5}=-\ell-\delta-\mu$.

Here, $\lambda_{1}, \lambda_{2}, \lambda_{3}$ and $\lambda_{5}$ are clearly real and negative. Also as $R_{0}<1$, then

$$
\beta S_{c}^{0}+\alpha_{2} S_{d}^{0}<\gamma+\mu+\mu_{1}
$$

and therefore $\lambda_{4}$ is also real and negative. Hence, the system (1) shows local asymptotic stability at $E^{(0)}$.

Theorem 5 The disease-free equilibrium $E^{(0)}$ is globally asymptotically stable in $R^{5}$ if $R_{0}<1$. 
Proof We construct a Lyapunov function $L\left(S_{d}, D, S_{c}, I_{c}, R_{c}\right): R^{5} \rightarrow R^{+}$as

$$
L\left(S_{d}, D, S_{c}, I_{c}, R_{c}\right)=h I_{c}
$$

for every $h \geq 0$ for the disease-free equilibrium $E^{(0)}=\left(S_{d}^{0}, 0, S_{c}^{0}, 0,0\right)=$ $\left(\frac{\Phi}{\alpha_{1}+\mu}, 0, \frac{\Lambda}{\mu}, 0,0\right)$.

Differentiating $L\left(S_{d}, D, S_{c}, I_{c}, R_{c}\right)$ with respect to times gives

$$
\frac{d L}{d t}\left(S_{d}, D, S_{c}, I_{c}, R_{c}\right)=h \frac{d I_{c}}{d t}
$$

Substituting the equation $\frac{d I_{c}}{d t}$, we get

$$
\frac{d L}{d t}\left(S_{d}, D, S_{c}, I_{c}, R_{c}\right)=h\left[\beta S_{c} I_{c}+\rho D I_{c}+\alpha_{2} S_{d} I_{c}-\gamma I_{c}-\mu I_{c}-\mu_{1} I_{c}\right]
$$

Since $S_{d}=\frac{\Phi}{\alpha_{1}+\mu}, S_{c}=\frac{\Lambda}{\mu}$ and $D=0$ at $t_{0}$ we have,

$$
\begin{array}{r}
\Longrightarrow h\left(\gamma+\mu+\mu_{1}\right)\left[\frac{\beta \Lambda\left(\alpha_{1}+\mu\right)+\alpha_{2} \Phi \mu}{\mu\left(\alpha_{1}+\mu\right)\left(\gamma+\mu+\mu_{1}\right)}-1\right] I_{c} \\
\Longrightarrow h\left(\gamma+\mu+\mu_{1}\right)\left[R_{0}-1\right] I_{c}
\end{array}
$$

where $R_{0}=\frac{\beta \Lambda\left(\alpha_{1}+\mu\right)+\alpha_{2} \Phi \mu}{\mu\left(\alpha_{1}+\mu\right)\left(\gamma+\mu+\mu_{1}\right)}$ and $h=\frac{1}{\left(\gamma+\mu+\mu_{1}\right)}$

$$
\frac{d L}{d t}\left(S_{d}, D, S_{c}, I_{c}, R_{c}\right)=\left[R_{0}-1\right] I_{c} \leq 0
$$

From (5), when $I_{c}=0, \frac{d L}{d t}\left(S_{d}, D, S_{c}, I_{c}, R_{c}\right)=0$, subsituting $I_{c}=0$ into equation (1) for $\frac{d S_{d}}{d t} \rightarrow \frac{\Phi}{\alpha_{1}+\mu}, \frac{d S_{c}}{d t} \rightarrow \frac{\Lambda}{\mu}$ and $D, R_{c} \rightarrow 0$ as $T \rightarrow \infty$. It follows that, the maximum invariant set in $\left\{\left(S_{d}, D, S_{c}, I_{c}, R_{c}\right) \epsilon \frac{d L}{d t}\left(S_{d}, D, S_{c}, I_{c}, R_{c}\right) \leq 0\right\}$ is the singleton set $\left\{E^{(0)}\right\}$

Theorem 6 The endemic equilibrium $E^{(1)}=\left(S_{d}^{*}, D^{*}, S_{c}^{*}, I_{c}^{*}, R_{c}^{*}\right)$ of the system (1) is locally asymptotically stable if and only if following conditions hold:

i. $a_{i}>0 \quad i=1,2,3,4,5$

ii. $a_{1} a_{2} a_{3}>a_{3}^{2}+a_{1}^{2} a_{4}$

iii. $\left(a_{1} a_{4}-a_{5}\right)\left(a_{1} a_{2} a_{3}-a_{3}^{2}-a_{1}^{2} a_{4}\right)>a_{5}\left(a_{1} a_{2}-a_{3}\right)^{2}+a_{1} a_{5}^{2}$

Proof The variational matrix evaluated at the equilibrium point $E^{(1)}$ is given by

$$
M^{*}=\left(\begin{array}{ccccc}
a_{11} & 0 & 0 & a_{14} & 0 \\
a_{21} & a_{22} & 0 & a_{24} & a_{25} \\
0 & 0 & a_{33} & a_{34} & a_{35} \\
a_{41} & a_{42} & a_{43} & a_{44} & 0 \\
0 & 0 & 0 & a_{54} & a_{55}
\end{array}\right)
$$


where,

$$
\begin{aligned}
& a_{11}=-\alpha_{1}-\alpha_{2} I_{c}^{*}-\mu, \quad a_{14}=\alpha_{2} S_{d}^{*} \\
& a_{21}=\alpha_{1}, \quad a_{22}=-\rho I_{c}^{*}-\mu, \quad a_{24}=-\rho D^{*}, \quad a_{25}=\delta \\
& a_{33}=-\beta I_{c}^{*}-\mu, \quad a_{34}=-\beta S_{c} *, a_{35}=\ell \\
& a_{41}=\alpha_{2} I_{c}^{*}, \quad a_{42}=\rho I_{c}^{*}, \quad a_{43}=\beta I_{c}^{*}, \quad a_{44}=\beta S_{c}^{*}+\rho D^{*}+\alpha_{2} S_{d}^{*}-\gamma-\mu-\mu_{1} \\
& a_{54}=\gamma a_{55}=-\ell-\delta-\mu
\end{aligned}
$$

The characteristic equation corresponding to this variational matrix is given by the following polynomial equation is

$$
\lambda^{5}+a_{1} \lambda^{4}+a_{2} \lambda^{3}+a_{3} \lambda^{2}+a_{4} \lambda+a_{5}=0
$$

where

$$
\begin{aligned}
a_{1}= & -\left(a_{11}+a_{22}+a_{33}+a_{44}+a_{55}\right) \\
a_{2}= & \left(a_{44} a_{55}+a_{33} a_{55}+a_{11} a_{55}+a_{22} a_{55}+a_{33} a_{44}+a_{11} a_{44}+a_{22} a_{44}+a_{11} a_{33}+\right. \\
& \left.a_{22} a_{33}+a_{11} a_{22}-a_{34} a_{43}-a_{24} a_{42}-a_{14} a_{41}\right) \\
a_{3}= & -\left(a_{33} a_{44} a_{55}+a_{11} a_{44} a_{55}+a_{22} a_{44} a_{55}+a_{11} a_{33} a_{55}+a_{22} a_{33} a_{55}+a_{11} a_{22} a_{55}+a_{11} a_{33} a_{44}\right. \\
& +a_{22} a_{33} a_{44}+a_{11} a_{22} a_{44}+a_{11} a_{22} a_{33}-a_{34} a_{43} a_{55}-a_{34} a_{43} a_{11}-a_{34} a_{43} a_{22}+a_{35} a_{43} a_{54}- \\
& \left.a_{24} a_{42} a_{55}-a_{24} a_{42} a_{11}-a_{24} a_{42} a_{33}+a_{25} a_{54} a_{42}+a_{14} a_{21} a_{42}-a_{14} a_{41} a_{55}-a_{14} a_{41} a_{22}-a_{14} a_{41} a_{33}\right) \\
a_{4}= & \left(a_{11} a_{33} a_{44} a_{55}+a_{22} a_{33} a_{44} a_{55}+a_{11} a_{22} a_{44} a_{55}+a_{11} a_{22} a_{33} a_{55}+a_{11} a_{22} a_{33} a_{44}-a_{34} a_{43} a_{11} a_{55}\right. \\
& -a_{34} a_{43} a_{22} a_{55}-a_{34} a_{43} a_{11} a_{22}+a_{11} a_{35} a_{43} a_{54}+a_{22} a_{35} a_{43} a_{54}-a_{24} a_{42} a_{11} a_{55}-a_{24} a_{42} a_{33} a_{55} \\
& -a_{24} a_{42} a_{11} a_{33}+a_{25} a_{54} a_{42} a_{11}+a_{25} a_{54} a_{42} a_{33}+a_{14} a_{21} a_{42} a_{33}+a_{14} a_{21} a_{42} a_{55}-a_{14} a_{41} a_{22} a_{55} \\
& \left.-a_{14} a_{41} a_{33} a_{55}-a_{14} a_{41} a_{22} a_{33}\right) \\
a_{5}= & -\left(a_{11} a_{22} a_{33} a_{44} a_{55}-a_{34} a_{43} a_{11} a_{22} a_{55}+a_{11} a_{22} a_{35} a_{43} a_{54}-a_{42} a_{24} a_{11} a_{33} a_{55}+a_{25} a_{54} a_{42} a_{11} a_{33}\right. \\
& \left.+a_{14} a_{21} a_{42} a_{33} a_{55}-a_{14} a_{41} a_{22} a_{33} a_{55}\right)
\end{aligned}
$$

By Routh-Hurwitz criteria, $E^{(1)}$ will be locally asymptotically stable if the following conditions are satisfied :

- $a_{i}>0 \quad i=1,2,3,4,5$

$-a_{1} a_{2} a_{3}>a_{3}^{2}+a_{1}^{2} a_{4}$

- $\left(a_{1} a_{4}-a_{5}\right)\left(a_{1} a_{2} a_{3}-a_{3}^{2}-a_{1}^{2} a_{4}\right)>a_{5}\left(a_{1} a_{2}-a_{3}\right)^{2}+a_{1} a_{5}^{2}$ 


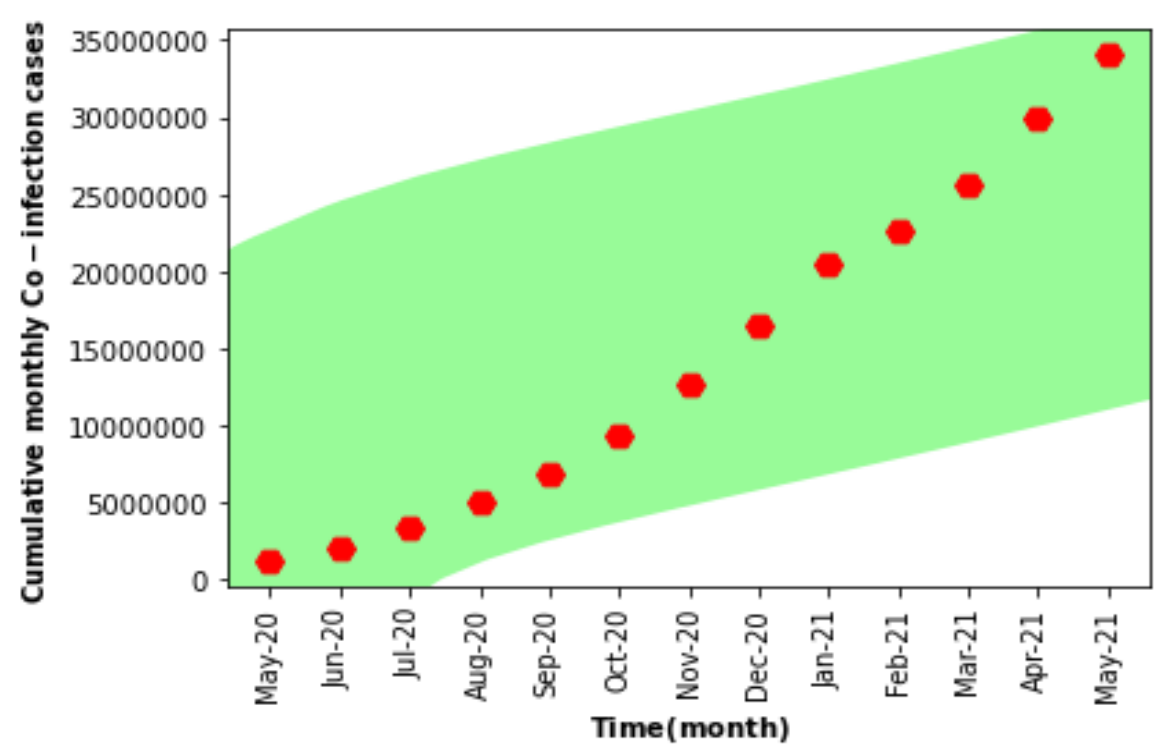

Fig. 8: Plots of the output of the fitted model (1) and the observed cases during May2020 to May-2021. Red dotted line shows real data points and shaded regions stands for model solution.

\section{Data fitting}

There may be some real situations to adopt a mathematical model that can be found in society. The same kind of adoption we are also trying to progress and corroborate with our results along with real data. On this aspect, we have absorbed the data of COVID19 confirmed cases as given in [1] which is worldwide during the time period May2020 to May-2021. Even though there may be some studies about diabetes patients having COVID-19, there is no availability of data about the number of COVID-19 patients who have diabetes.

It has been predicted that $15 \%-30 \%$ of COVID-19 patients have diabetes in some of the literature [53], [54], [55]. A report [56] from the Washington State demonstrates the fact that 33\% of COVID-19 patients had known diabetes. In Satman et. al. [57], the authors conducted a study based on the mortality rates of COVID-19 patients with and without type-2 diabetes in Istanbul, Turkey. Their analysis reveals that nearly $22 \%$ of COVID-19 patients have pre-existing diabetes. However, the findings of Chen et. al. [58] showed that 15\% had diabetes among 904 patients who have been hospitalized with a clinical diagnosis of infection.

Based on the above facts and data, we are also trying to follow that and consequently, we assume that $20 \%$ of the COVID-19 affected population reported by WHO have diabetes. Here we are using the reported cases (May-2020 to May-2021) of coinfection in six countries to calibrate the model (1). These data are taken from WHO [1]. 


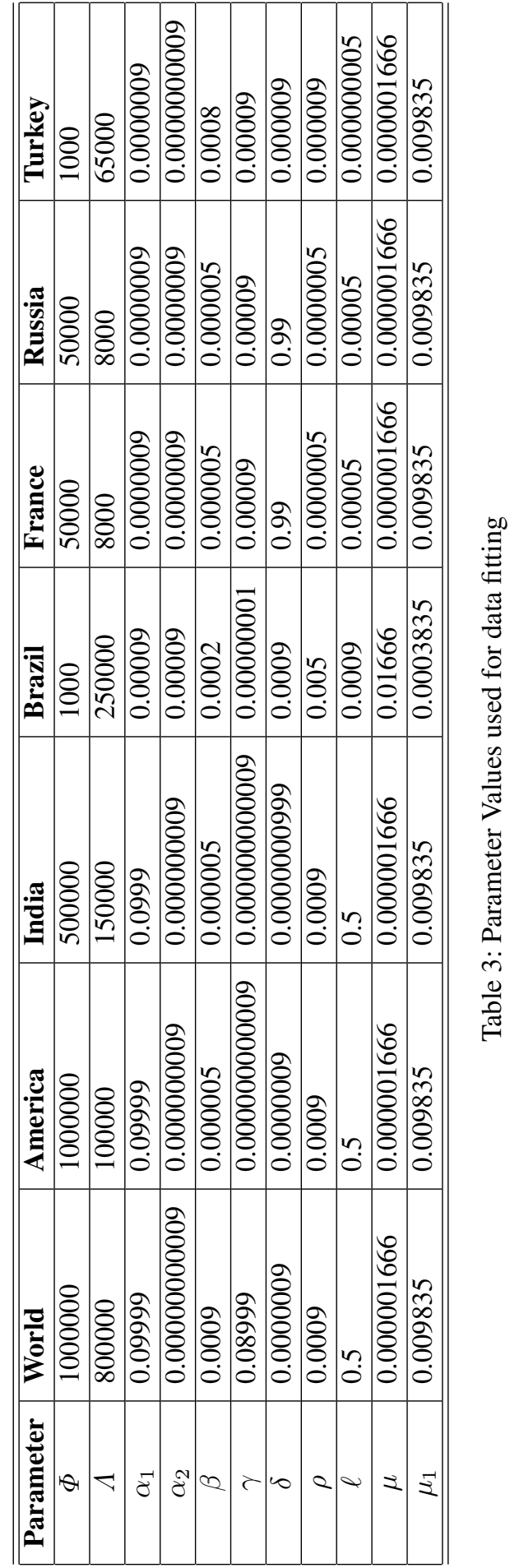



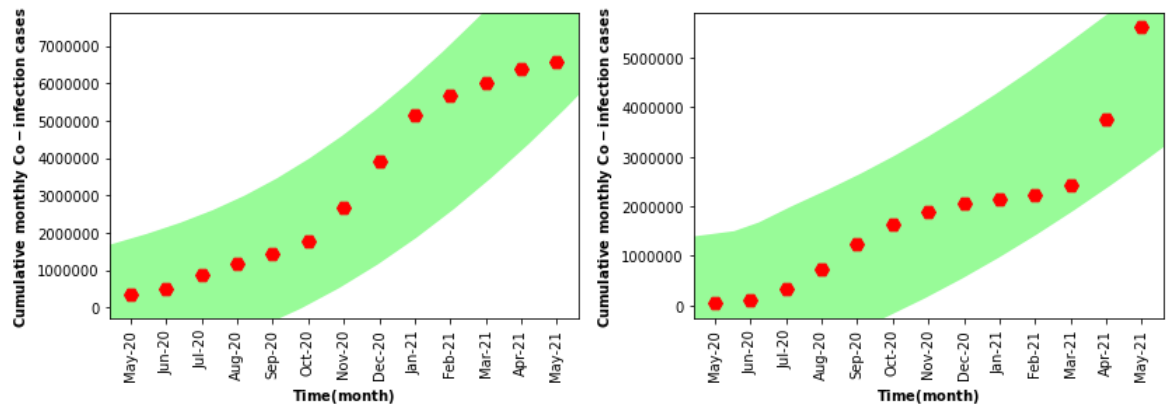

(a) America

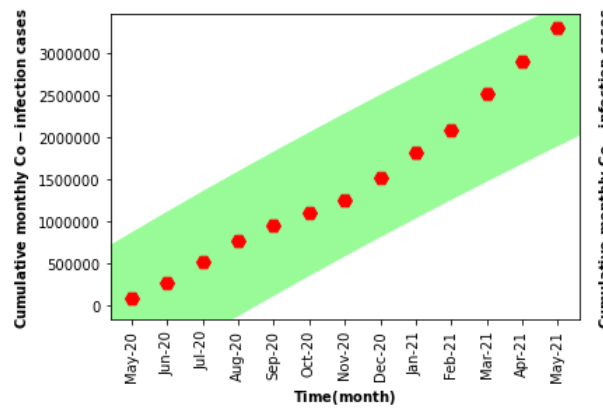

(c) Brazil

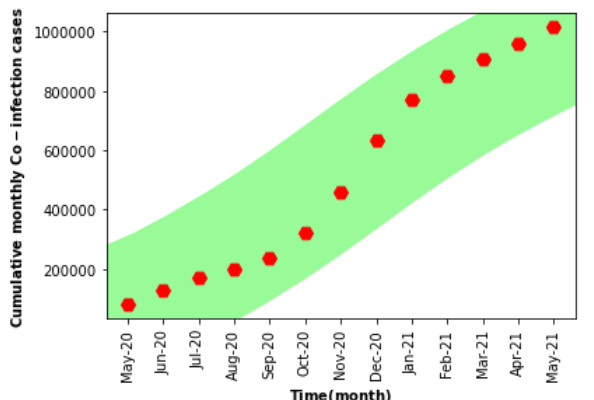

(e) Russia (b) India

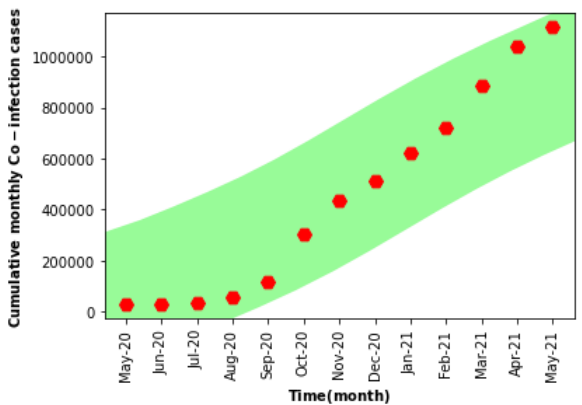

(d) France

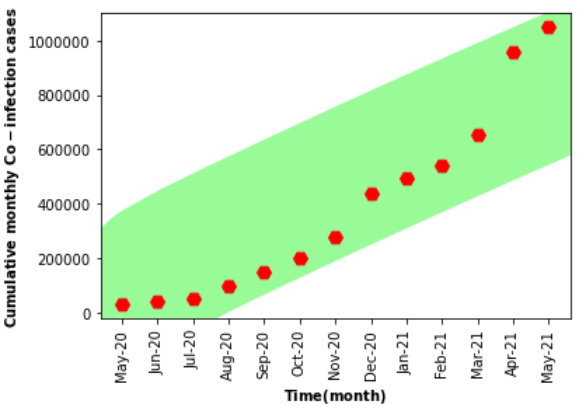

(f) Turkey

Fig. 9: Plots of the output of the fitted model (1) and the observed cases during May2020 to May-2021. Red dotted line shows real data points and shaded regions stands for model solution. 


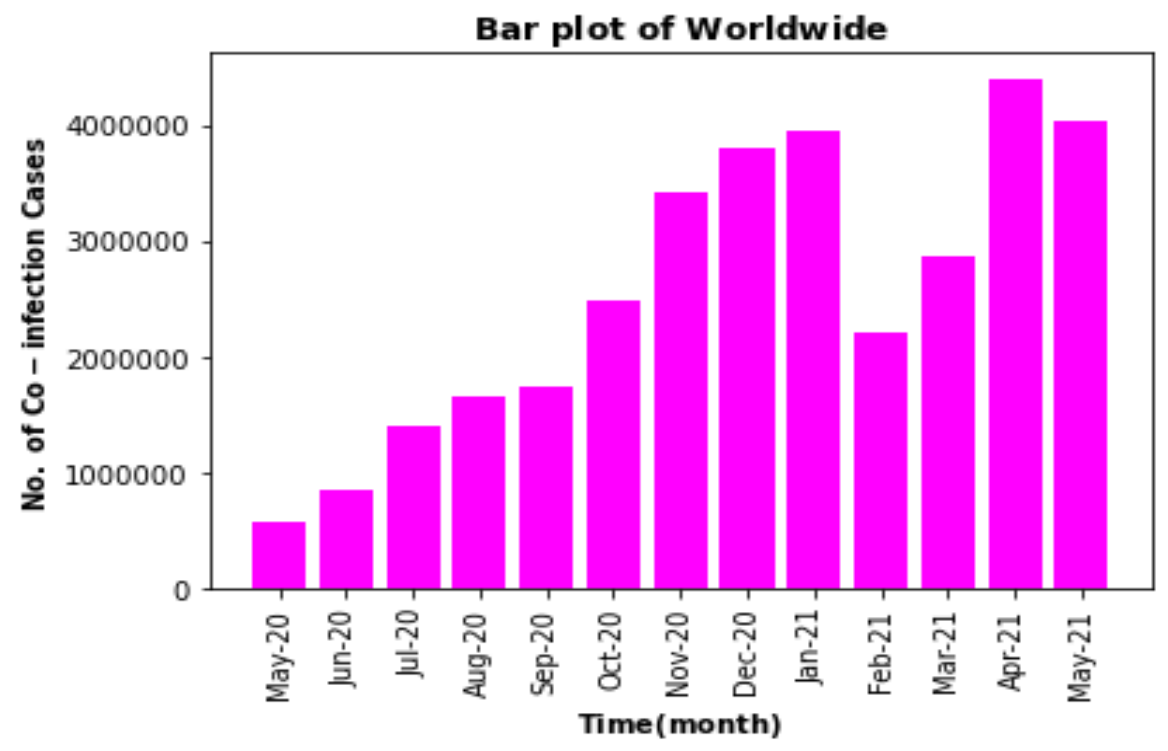

Fig. 10: Monthly Coinfection of Diabetes and COVID-19 cases for worldwide during May-2020 to May-2021.

For our study, we choose America [8], India [9], Brazil [10], France [11], Russia [12] and Turkey [13] as these are highly affected countries by COVID-19. Out of all these six countries, we can see that the highest number of cases are in America and less number of cases are in Turkey. Fitted data with a model solution is shown in Fig. 8.9.

The comparison of our model (1) and real data, during the period from May-2020 to May-2021 presented below. Fig. 8 represents a comparison of real data and our model for co-infection cases from May-2020 to May-2021. The simulated results are close to the real data in co-infection cases as depicted in Fig. 8, And in Fig. 9, fitted cumulative cases for America, India, Brazil, France, Russia, and Turkey, are shown respectively.

From the data of cumulative confirmed cases, we calculate the month-wise data. The monthly confirmed cases of co-infection worldwide during the period of study are provided in Fig. 10 From Fig. 11 we can see the scenario of monthly co-infection cases for different countries namely, America, India, Brazil, France, Russia, and Turkey, respectively.

\section{Stochastic model}

Here, we extend our deterministic model (1) to stochastic model as stochastic models are more capable of capturing random variations in biological dynamics of the problem under consideration. The derivation of the stochastic model is based on the 


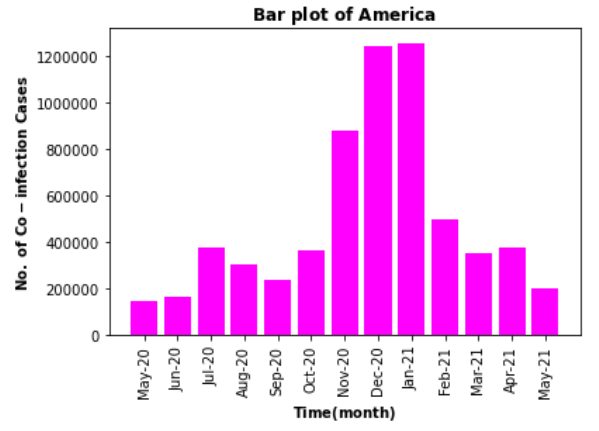

(a) America

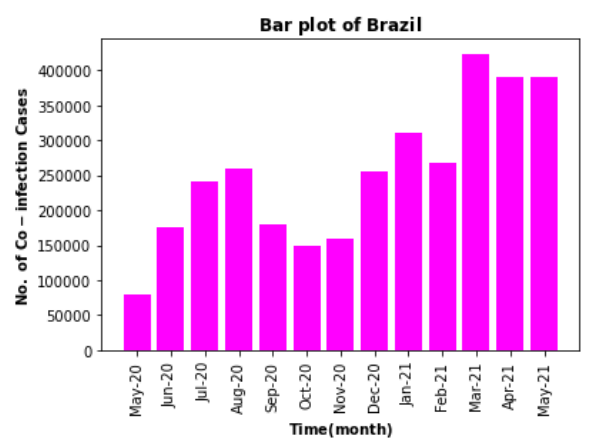

(c) Brazil

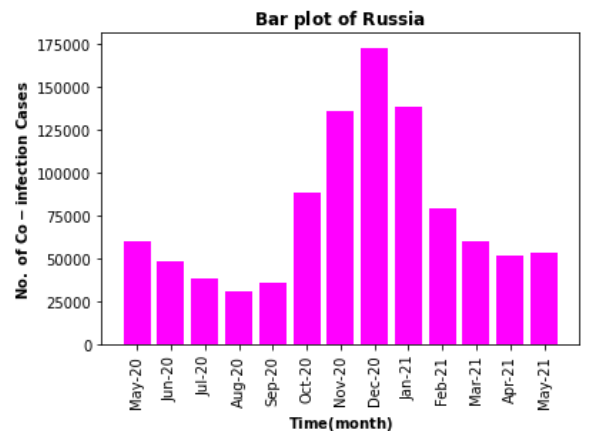

(e) Russia

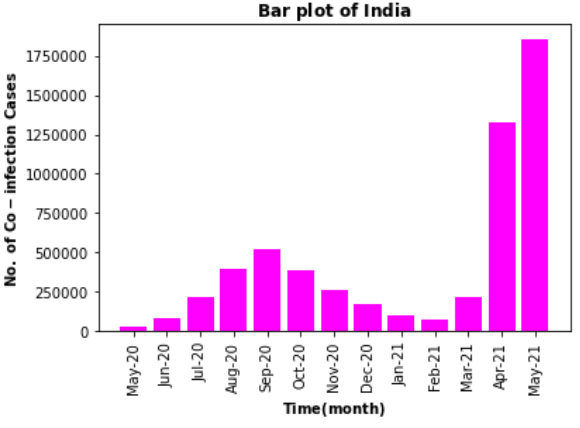

(b) India

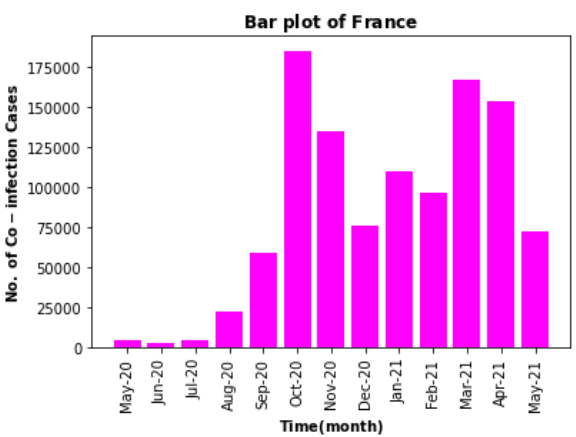

(d) France

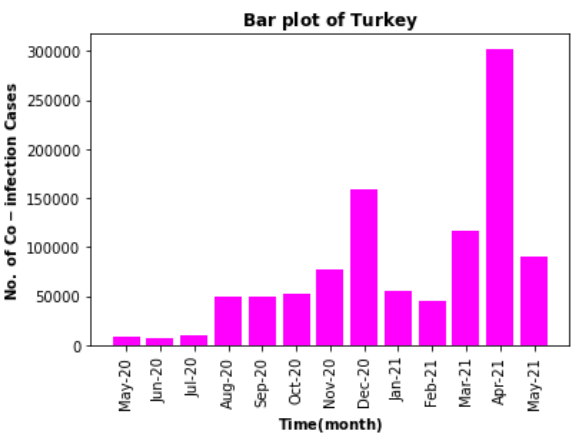

(f) Turkey

Fig. 11: Monthly Coinfection of Diabetes and COVID-19 cases for different countries from May-2020 to May-2021 
Table 4: Possible changes of states and their probabilities.

\begin{tabular}{lll}
\hline Possible stage change & & Probability of state changes \\
\hline$(\Delta Z)_{1}$ & $=(1,0,0,0,0)^{H}$ & $P_{1}=\Phi \Delta t+o(\Delta t)$ \\
$(\Delta Z)_{2}$ & $=(-1,1,0,0,0)^{H}$ & $P_{2}=\alpha_{1} Z_{1} \Delta t+o(\Delta t)$ \\
$(\Delta Z)_{3}$ & $=(-1,0,0,1,0)^{H}$ & $P_{3}=\alpha_{2} Z_{1} Z_{4} \Delta t+o(\Delta t)$ \\
$(\Delta Z)_{4}$ & $=(-1,0,0,0,0)^{H}$ & $P_{4}=\mu Z_{1} \Delta t+o(\Delta t)$ \\
$(\Delta Z)_{5}$ & $=(0,1,0,0,-1)^{H}$ & $P_{5}=\delta Z_{5} \Delta t+o(\Delta t)$ \\
$(\Delta Z)_{6}$ & $=(0,-1,0,1,0)^{H}$ & $P_{6}=\rho Z_{2} Z_{4} \Delta t+o(\Delta t)$ \\
$(\Delta Z)_{7}$ & $=(0,-1,0,0,0)^{H}$ & $P_{7}=\mu Z_{2} \Delta t+o(\Delta t)$ \\
$(\Delta Z)_{8}$ & $=(0,0,1,0,0)^{H}$ & $P_{8}=\Lambda \Delta t+o(\Delta t)$ \\
$(\Delta Z)_{9}$ & $=(0,0,1,0,-1)^{H}$ & $P_{9}=\ell Z_{5} \Delta t+o(\Delta t)$ \\
$(\Delta Z)_{10}$ & $=(0,0,-1,1,0)^{H}$ & $P_{10}=\beta Z_{3} Z_{4} \Delta t+o(\Delta t)$ \\
$(\Delta Z)_{11}$ & $=(0,0,-1,0,0)^{H}$ & $P_{11}=\mu Z_{3} \Delta t+o(\Delta t)$ \\
$(\Delta Z)_{12}$ & $=(0,0,0,-1,1)^{H}$ & $P_{12}=\gamma Z_{4} \Delta t+o(\Delta t)$ \\
$(\Delta Z)_{13}$ & $=(0,0,0,-1,0)^{H}$ & $P_{13}=\mu Z_{4} \Delta t+o(\Delta t)$ \\
$(\Delta Z)_{14}$ & $=(0,0,0,-1,0)^{H}$ & $P_{14}=\mu_{1} Z_{4} \Delta t+o(\Delta t)$ \\
$(\Delta Z)_{15}$ & $=(0,0,0,0,-1)^{H}$ & $P_{15}=\mu Z_{5} \Delta t+o(\Delta t)$ \\
$(\Delta Z)_{16}$ & $=(0,0,0,0,0)^{H}$ & $P_{16}=1-\sum_{i=1}^{15} P_{i} \Delta t+o(\Delta t)$ \\
\hline
\end{tabular}

method developed by Yuan et al. [59]. Let $Z(t)=\left(Z_{1}(t), Z_{2}(t), Z_{3}(t), Z_{4}(t), Z_{5}(t)\right)^{H}$ be a continuous random variable for $\left(S_{d}(t), D(t), S_{c}(t), I_{c}(t), R_{c}(t)\right)^{H}$ and $H$ denotes the transpose of matrix.

Let $\Delta Z=Z(t+\Delta t)-Z(t)=\left(\Delta Z_{1}, \Delta Z_{2}, \Delta Z_{3}, \Delta Z_{4}, \Delta Z_{5}\right)^{H}$ denote the random vector for the change in random variables during time interval $\Delta t$. Here we will write the transition maps which define all possible changes between states in the stochastic model. On the basis of our deterministic model (1), here we see that there exist 16 possible changes between states in a small-time interval $\Delta t$. State changes and their probabilities are discussed in Table 4 .

Let us consider the case when one susceptible human becomes infected human due to the imbalanced level of glucose. In this case the state change $\Delta Z$ is denoted by $\Delta Z=(-1,1,0,0,0)$ its probability of the change is given by

$\operatorname{Prob}\left\{\left(\Delta Z_{1}, \Delta Z_{2}, \Delta Z_{3}, \Delta Z_{4}, \Delta Z_{5}\right)=(-1,1,0,0,0) \mid\left(Z_{1}, Z_{2}, Z_{3}, Z_{4}, Z_{5}\right)\right\}=P_{2}=\alpha_{1} Z_{1} Z_{2} \Delta t+o(\Delta t)$.

One can easily determine the expectation change $E(\Delta Z)$ and its covariance matrix $V(\Delta Z)$ associated with $\Delta Z$ by neglecting the terms higher than $o(\Delta t)$. The expectation of $\Delta Z$ is given by 


$$
\begin{aligned}
E(\Delta Z)=\sum_{i=1}^{15} P_{i}(\Delta Z)_{i} \Delta t & =\left(\begin{array}{c}
\Phi-\alpha_{1} Z_{1}-\alpha_{2} Z_{1} Z_{4}-\mu Z_{1} \\
\alpha_{1} Z_{1}+\delta Z_{5}-\rho Z_{2} Z_{4}-\mu Z_{2} \\
\Lambda+\ell Z_{5}-\beta Z_{3} Z_{4}-\mu Z_{3} \\
\beta Z_{3} Z_{4}+\rho Z_{2} Z_{4}+\alpha_{2} Z_{1} Z_{4}-\gamma Z_{4}-\mu Z_{4}-\mu_{1} Z_{4} \\
\gamma Z_{4}-\ell Z_{5}-\delta Z_{5}-\mu Z_{5}
\end{array}\right) \Delta t \\
& =f\left(Z_{1}, Z_{2}, Z_{3}, Z_{4}, Z_{5}\right) \Delta t .
\end{aligned}
$$

Here it can be noted that the expectation vector and the function $f$ are in the same form as those in deterministic model (1). Since the covariance matrix $V(\Delta Z)=$ $E\left((\Delta Z)(\Delta Z)^{H}\right)-E(\Delta Z)\left(E(\Delta Z)^{H}\right)$ and $E\left((\Delta Z)(\Delta Z)^{H}\right)=f(Z)\left(f(Z)^{H}\right) \Delta t$, it can be approximated with diffusion matrix $\Omega$ times $\Delta t$ by neglecting the term of $(\Delta t)^{2}$ such that $V(\Delta Z) \approx E\left((\Delta Z)(\Delta Z)^{H}\right)$.

Hence

$E\left((\Delta Z)(\Delta Z)^{H}\right)=\sum_{i=1}^{15} P_{i}\left((\Delta Z)_{i}(\Delta Z)_{i}^{H}\right) \Delta t=\left(\begin{array}{ccccc}V_{11} & V_{12} & 0 & V_{14} & 0 \\ V_{21} & V_{22} & 0 & V_{24} & V_{25} \\ 0 & 0 & V_{33} & V_{34} & V_{35} \\ V_{41} & V_{42} & V_{43} & V_{44} & V_{45} \\ 0 & V_{52} & V_{53} & V_{54} & V_{55}\end{array}\right) . \Delta t=\Omega . \Delta t$

where the above diffusion matrix is symmetric, positive-definite and each component of this $5 \times 5$ diffusion matrix can be obtained as follows:

$$
\begin{aligned}
& V_{11}=P_{1}+P_{2}+P_{3}+P_{4}=\Phi+\alpha_{1} Z_{1}+\alpha_{2} Z_{1} Z_{4}+\mu Z_{1}, \\
& V_{22}=P_{2}+P_{5}+P_{6}+P_{7}=\alpha_{1} Z_{1}+\delta Z_{5}+\rho Z_{2} Z_{4}+\mu Z_{2}, \\
& V_{33}=P_{8}+P_{9}+P_{10}+P_{11}=\Lambda+\ell Z_{5}+\beta Z_{3} Z_{4}+\mu Z_{3}, \\
& V_{44}=P_{3}+P_{6}+P_{10}+P_{12}+P_{13}+P_{14}=\beta Z_{3} Z_{4}+\rho Z_{2} Z_{4}+\alpha_{2} Z_{1} Z_{4}+\gamma Z_{4}+\mu Z_{4}+\mu_{1} Z_{4}, \\
& V_{55}=P_{5}+P_{9}+P_{12}+P_{15}=\gamma Z_{4}+\ell Z_{5}+\delta Z_{5}+\mu Z_{5}, \\
& V_{12}=V_{21}=-P_{2}=-\alpha_{1} Z_{1}, \\
& V_{14}=V_{41}=-P_{3}=-\alpha_{2} Z_{1} Z_{4}, \\
& V_{24}=V_{42}=-P_{6}=-\rho Z_{2} Z_{4}, \\
& V_{25}=V_{52}=-P_{5}=-\delta Z_{5}, \\
& V_{34}=V_{43}=-P_{10}=-\beta Z_{3} Z_{4}, \\
& V_{35}=V_{53}=-P_{9}=-\ell Z_{5}, \\
& V_{45}=V_{54}=-P_{12}=-\gamma Z_{4} .
\end{aligned}
$$

We follow the approach discussed in [59] and construct a matrix $Q$ such that $\Omega=Q Q^{H}$, where $Q$ is a $5 \times 12$ matrix. 


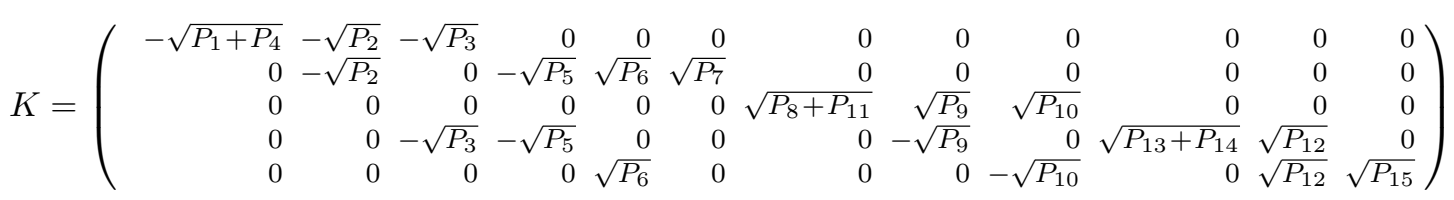

Then, the Ito stochastic differential model has the following form:

$$
d(Z(t))=f\left(Z_{1}, Z_{2}, Z_{3}, Z_{4}, Z_{5}\right) d t+Q \cdot d W(t)
$$

with initial condition $Z(0)=\left(Z_{1}(0), Z_{2}(0), Z_{3}(0), Z_{4}(0), Z_{5}(0)\right)^{H}$ and a Wiener process,

$W(t)=\left(W_{1}(t), W_{2}(t), W_{3}(t), W_{4}(t), W_{5}(t), W_{6}(t), W_{7}(t), W_{8}(t), W_{9}(t), W_{10}(t), W_{11}(t), W_{12}(t)\right)^{H}$.

Keeping in view of the above facts, we construct the stochastic differential equation model as follows:

$$
\begin{aligned}
\frac{d S_{d}}{d t}= & {\left[\Phi-\alpha_{1} S_{d}-\alpha_{2} S_{d} I_{c}-\mu S_{d}\right] d t+\sqrt{\Phi+\mu S_{d}} d W_{1}-\sqrt{\alpha_{1} S_{d}} d W_{2}-\sqrt{\alpha_{2} S_{d} I_{c}} d W_{3} } \\
\frac{d D}{d t}= & {\left[\alpha_{1} S_{d}+\delta R_{c}-\rho D I_{c}-\mu D\right] d t+\sqrt{\alpha_{1} S_{d}} d W_{2}-\sqrt{\rho D I_{c}} d W_{5}+\sqrt{\delta R_{c}} d W_{5}+\sqrt{\mu D} d W_{6} } \\
\frac{d S_{c}}{d t}= & {\left[\Lambda+\ell R_{c}-\beta S_{c} I_{c}-\mu S_{c}\right] d t+\sqrt{\Lambda+\mu S_{c}} d W_{7}+\sqrt{\beta S_{c} I_{c}} d W_{8}+\sqrt{\ell R_{c}} d W_{9} } \\
\frac{d I_{c}}{d t}= & {\left[\beta S_{c} I_{c}+\rho D I_{c}+\alpha_{2} S_{d} I_{c}-\gamma I_{c}-\mu I_{c}-\mu_{1} I_{c}\right] d t+\sqrt{\alpha_{2} S_{d} I_{c}} d W_{3}+\sqrt{\rho D I_{c}} d W_{4} } \\
& -\sqrt{\beta S_{c} I_{c}} d W_{8}+\sqrt{\mu_{1} I_{c}+\mu I_{c}} d W_{10}+\sqrt{\gamma I_{c}} d W_{11} \\
\frac{d R_{c}}{d t}= & {\left[\gamma I_{c}-\ell R_{c}-\delta R_{c}-\mu R_{c}\right] d t+\sqrt{\delta R_{c}} d W_{5}-\sqrt{\ell R_{c}} d W_{9}-\sqrt{\gamma I_{c}} d W_{11}+\sqrt{\mu R_{c}} d W_{12} }
\end{aligned}
$$

\section{Numerical simulation}

Analytical studies can never be definitive without numerical simulation. In this section, we have performed a numerical simulation to validate the analytical results obtained in the above sections. First, we consider the following set of parameters which corresponds to the DFE point. All parameters are in per year.

$$
\begin{aligned}
P_{1}= & \left(\Phi, \Lambda, \alpha_{1}, \alpha_{2}, \delta, \rho, \beta, \gamma, \ell, \mu, \mu_{1}\right) \\
= & (100,80,0.00008,0.000000999,0.1,0.0000009,0.000009,0.0007,0.008, \\
& 0.01666,0.03835)
\end{aligned}
$$

Using these parameter values and initial condition as $S_{d}(0)=1000, D(0)=$ $800, S_{c}(0)=900, I_{c}(0)=700, R_{c}(0)=600$ we get $R_{0}<1$ and the dynamics of the model (1) is exhibit in Fig. 12. This figure shows that Diabetes class $(D)$, COVID19 Susceptible class $\left(S_{c}\right)$, COVID-19 Infected class $\left(I_{c}\right)$ and COVID-19 Recovered $\operatorname{class}\left(R_{c}\right)$ decline to zero (extinct), i.e., the population approaches to the DFE $E^{(0)}=$ 


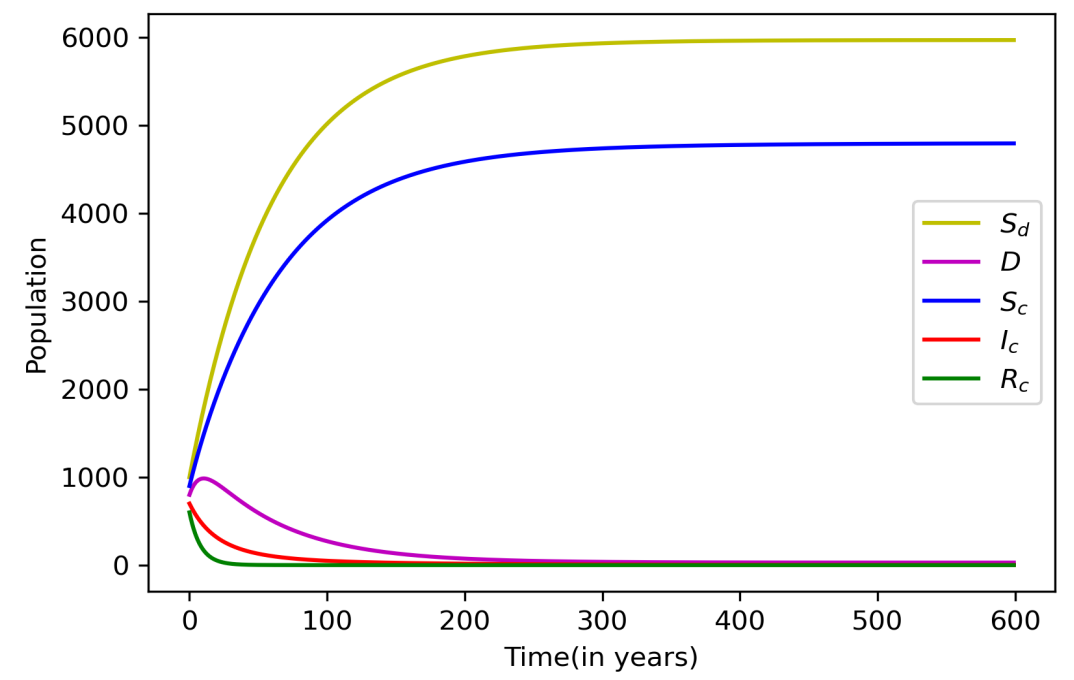

Fig. 12: Variation of $S_{d}, D, S_{c}, I_{c}, R_{c}$ with time showing the stability of disease free equilibrium point

$\left(S_{d}^{0}, 0, S_{c}^{0}, 0,0\right)=(5973,0,4801,0,0)$. Here, the condition of Theorem 5 is also satisfied and consequently $E^{(0)}$ is globally asymptotically stable.

Next, we choose the set of parameters for EE point

$$
\begin{aligned}
P_{2}= & \left(\Phi, \Lambda, \alpha_{1}, \alpha_{2}, \delta, \rho, \beta, \gamma, \ell, \mu, \mu_{1}\right) \\
= & (1000,500,0.009,0.9,0.000000000789,0.000005,0.000000000298,0.009 \\
& 0.000000008,0.01666,0.03835)
\end{aligned}
$$

Using these parameter values and initial condition as $S_{d}(0)=10000, D(0)=$ $9000, S_{c}(0)=10000, I_{c}(0)=8000, R_{c}(0)=4000$ we get $R_{0}>1$ and the dynamics of the model (1) is demonstrated in Fig. 13. This figure shows that Diabetes susceptible class $\left(S_{d}\right)$, Diabetes class $(D)$, COVID-19 Susceptible class $\left(S_{c}\right)$, COVID-19 Infected class $\left(I_{c}\right)$ and COVID-19 Recovered class $\left(R_{c}\right)$ all persist, i.e., the population tends to EE point $E^{(1)}=\left(S_{d}^{*}, D^{*}, S_{c}^{*}, I_{c}^{*}, R_{c}^{*}\right)=(32889,6880,30008,5272,2848)$, which supports our mathematical result obtained in Theorem 6

Furthermore, we simulate the stochastic model 6 by using Euler-Maruyama method for the parameter set $P_{2}$. The simulation results for both models are shown in Fig. 14. We compare the mean of 100 runs of stochastic model simulation with the results of the corresponding deterministic model. In Fig. 15, the time series of all the variables of the model are plotted where the deterministic simulation is represented by a black dotted curve and the stochastic model is represented by colored curves. Here, it is clear that all classes in the stochastic simulation are very close to deterministic simulation. 


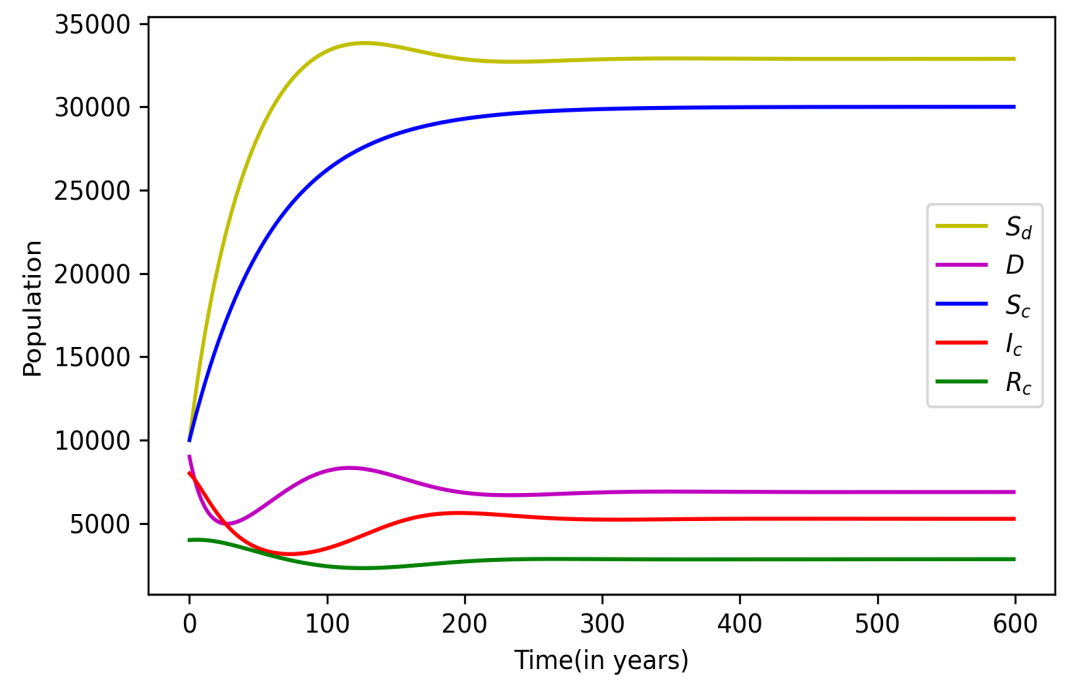

Fig. 13: Variation of $S_{d}, D, S_{c}, I_{c}, R_{c}$ with time showing the stability of endemic equilibrium point

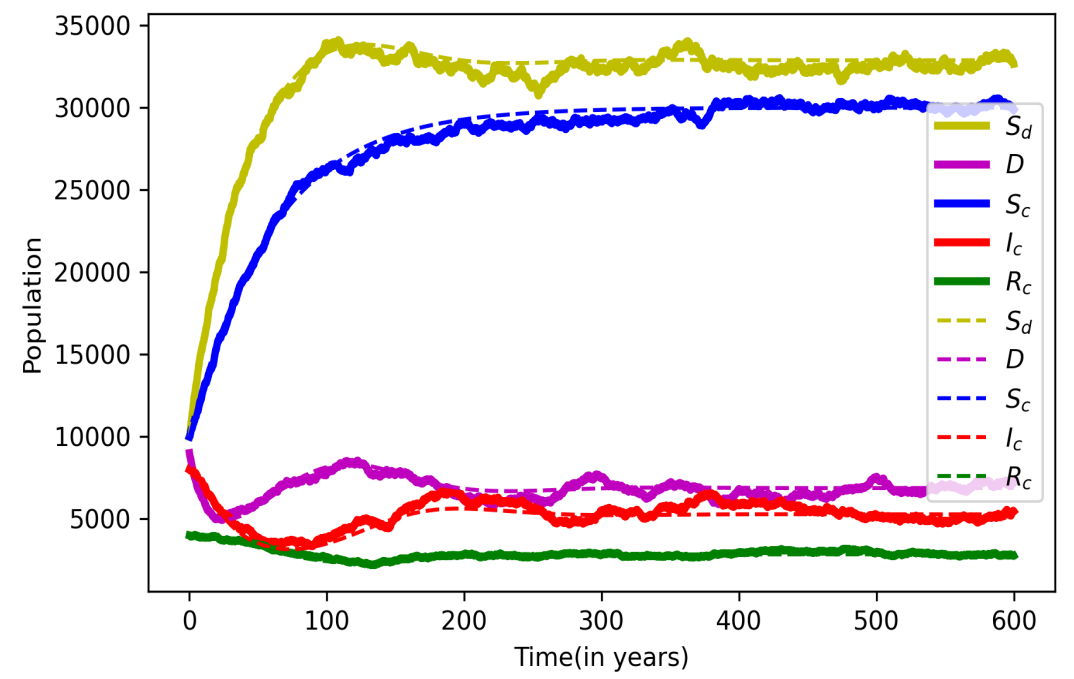

Fig. 14: Numerical simulation of the models (1) and (6) respectively at a time. 


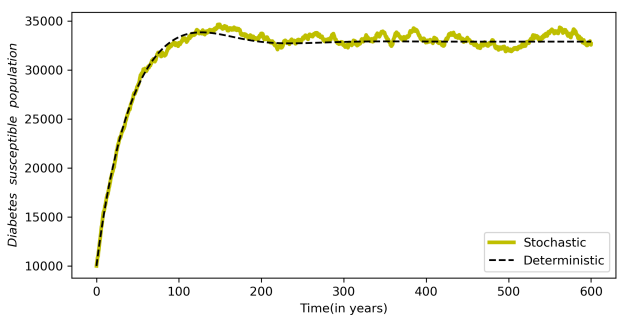

(a)

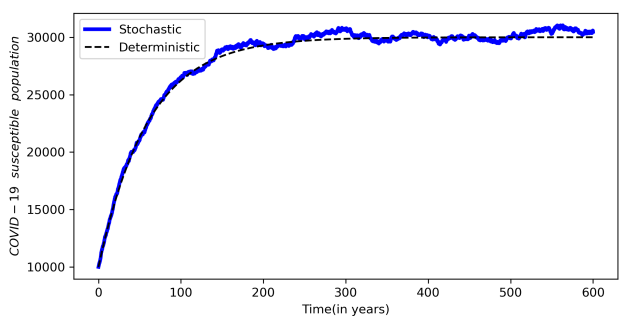

(c)

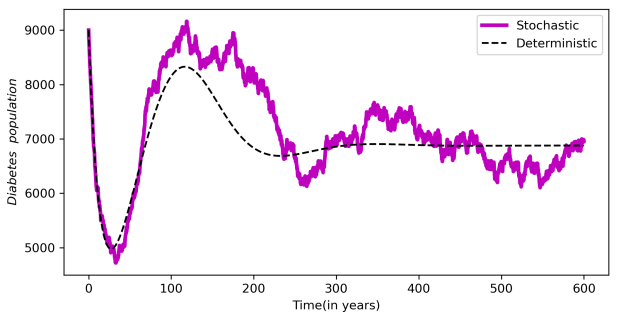

(b)

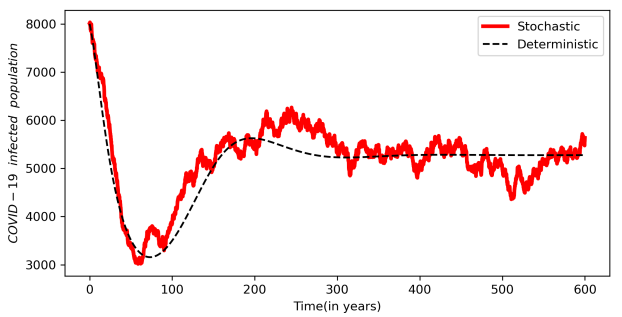

(d)

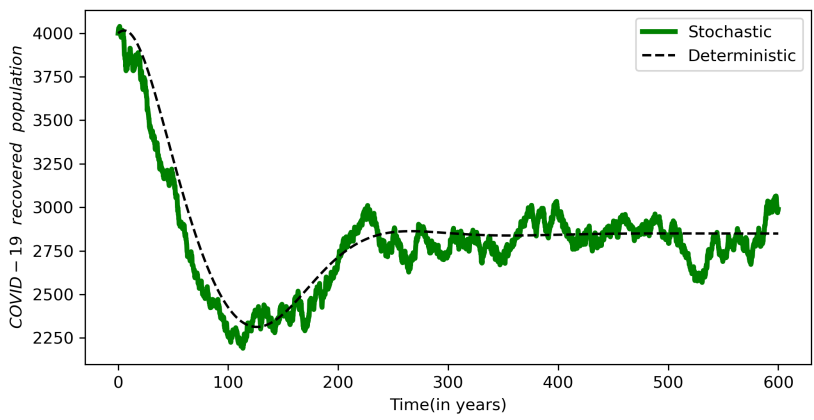

(e)

Fig. 15: Time evolution of (a) $S_{d}(t)$, (b) $D(t)$, (c) $S_{c}(t)$, (d) $I_{c}(t)$ and (e) $R_{c}(t)$ for the deterministic model (1) and stochastic model (6).

The impact of $\alpha_{1}$ and $\gamma$ on equilibrium levels of $S_{d}, D, I_{c}$ and $R_{c}$, are demonstrated in the Fig. 16-19. In Fig. 16 variation of $S_{d}$ with time is shown for different values of $\alpha_{1}$ by keeping all other parameters as in $P_{2}$. It is observed that with the increase in the value of $\alpha_{1}$ the equilibrium level of $S_{d}$ decreases.

The effect of the parameters $\alpha_{1}$ and $\gamma$ which corresponds to the transmission rate and recovery rate of diabetes population is demonstrated in Fig. $17 \mathrm{a}$ and $17 \mathrm{~b}$. From these figures, it is clear that with the increase in these parameters the diabetes population increases. 


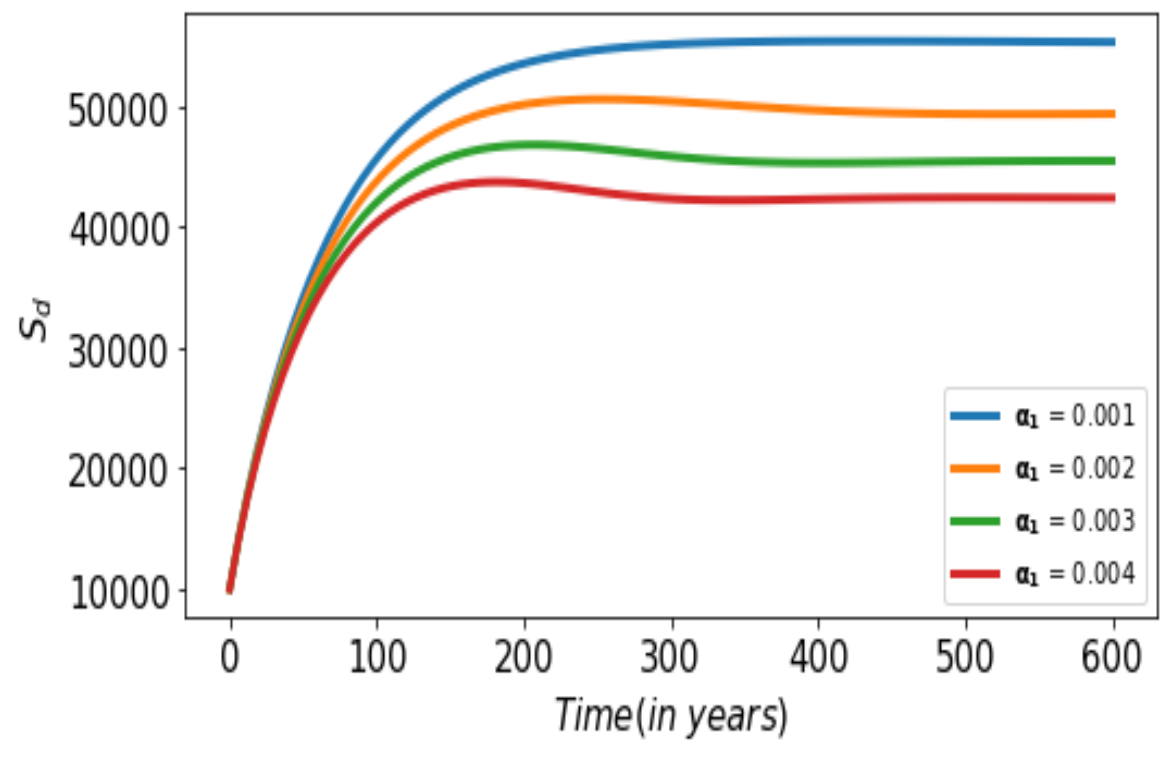

Fig. 16: Variation in diabetes susceptible population $\left(S_{d}\right)$ for different values of $\alpha_{1}$.

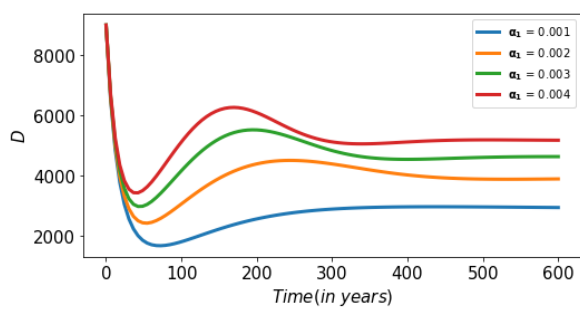

(a)

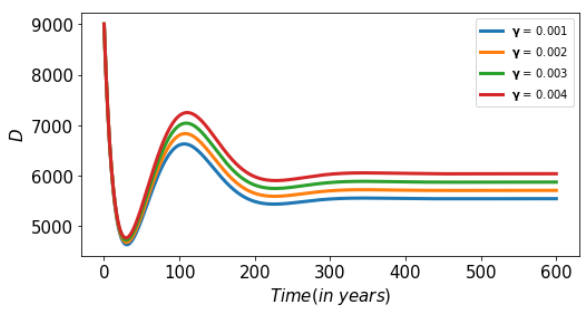

(b)

Fig. 17: Variation in diabetes population (D) for different values of $\alpha_{1}$ and $\gamma$.

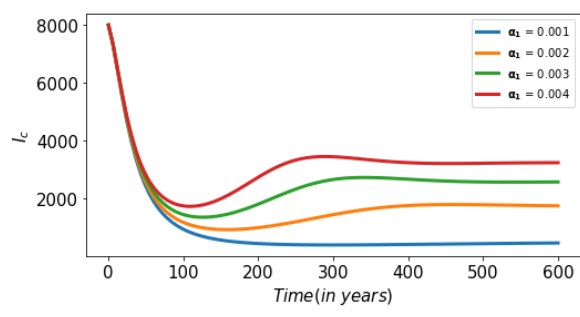

(a)

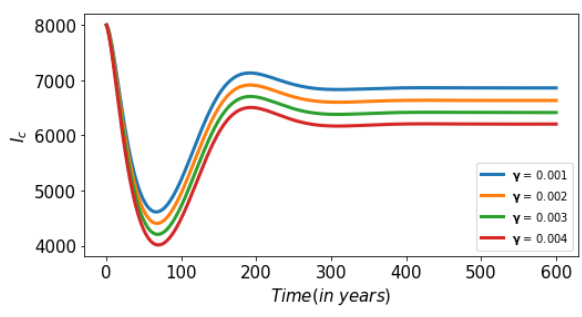

(b)

Fig. 18: Variation in COVID-19 infected population $\left(I_{c}\right)$ for different values of $\alpha_{1}$ and $\gamma$. 


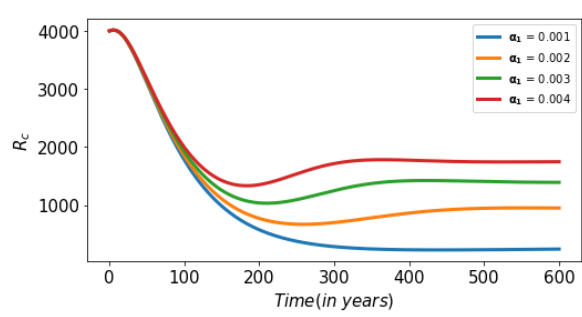

(a)

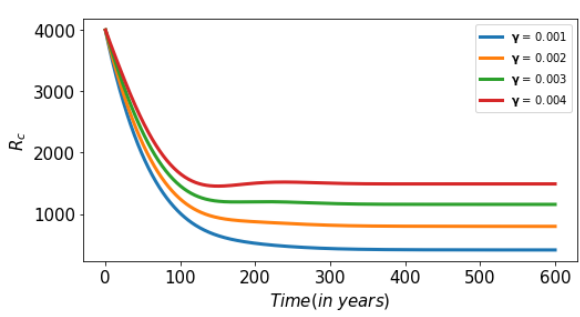

(b)

Fig. 19: Variation in COVID-19 recovered population $\left(R_{c}\right)$ for different values of $\alpha_{1}$ and $\gamma$.

In Fig. 18a, we see that COVID-19 infected population $\left(I_{c}\right)$ is directly proportional with the transmission rate $\left(\alpha_{1}\right)$. But $I_{c}$ is inversely proportional to the recovery rate of COVID-19 $(\gamma)$ is demonstrated in Fig. $18 \mathrm{~b}$.

In Fig. 19, the effects of COVID-19 recovered population $\left(R_{c}\right)$ is shown for different pairs of $\alpha_{1}$ and $\gamma$ by keeping all other parameters constant. From this plot, it is observed that the equilibrium levels of $R_{c}$ increase with the increase in the rates of $\alpha_{1}$ and $\gamma$.

\section{Result discussion and conclusion}

In this research, we have proposed a mathematical model for the co-infection of diabetes and COVID-19. Here, the population is divided into five compartments namely diabetes susceptible $\left(S_{d}\right)$, diabetes $(D)$, COVID-19 susceptible $\left(S_{c}\right)$, COVID-19 infected $\left(I_{c}\right)$ and COVID-19 recovered population $\left(R_{c}\right)$. First, the basic properties and existence of equilibrium points of the model (1) are discussed in detail. There exist two equillibria for our model: Disease Free Equilibrium(DFE) and Endemic Equilibrium(EE).

Then we computed the basic reproduction number $\left(R_{0}\right)$ by using the next-generation matrix method. Moreover, sensitivity analysis of $R_{0}$ is performed to find the significant parameters for the disease prevalence. It is observed that the transmission coefficient $\left(\alpha_{1}\right)$ from the diabetes susceptible class to diabetes class and recovery rate $(\gamma)$ plays a vital role in the reduction of reproduction number $R_{0}$. This fact is shown in Fig. 6-7.

Further, the stability of the different equilibria of the system is analyzed by using the stability theory of ordinary differential equations. The DFE point is globally asymptotically stable whenever $R_{0}<1$ and EE point is locally asymptotically stable under some restriction on parameters. Apart from these, the efficiency of our system is depicted through data fitting for the real data given by WHO [1].

For our study, we choose America, India, Brazil, France, Russia, and Turkey as these are most severely affected countries in the world. In Fig. 10-11 we can see the scenario of monthly diabetes and COVID-19 co-infection cases for worldwide and different countries namely, America, India, Brazil, France, Russia, and Turkey, 
respectively. And in Fig. 8- 9, fitted cumulative cases are illustrated. Our proposed model is fitted well with these real data sets. This fact clearly exhibits that our model suggesting a good amount of decrease compared with the actual projection by WHO.

Additionally, we extend our deterministic model (1) to stochastic model (6) and the simulation results of both the models are compared. It is observed that the stochastic simulation is almost close to the simulation results of the deterministic model (Fig. 14 .

Finally, to verify our analytical results, some numerical experimentation is provided. The effect of transmission coefficient $\left(\alpha_{1}\right)$ and recovery rate $(\gamma)$ on the equilibrium levels of $S_{d}, D, I_{c}$ and $R_{c}$ are illustrated in Fig. 16- 19 We have noted that the $\alpha_{1}$ and $\gamma$ are the key parameters and a small change in these parameters increases or decreases the COVID-19 infected population.

By observing all these results and analysis of our model the following facts have arrived.

1. Comorbidity disease increases the severity of the COVID-19 (i.e., increases the number of COVID-19 patients) and poses a significant threat to human life.

2. From the disease transmission rate and its effectiveness, we found that the progression towards COVID-19 is much higher for diabetes patients when compared with normal people.

3. By introducing our recovery rate due to COVID-19 medication reduces/eradicates the COVID-19 disease alone from individuals but diabetes patients will remain with diabetes as usual and continue the treatment/medication for diabetes.

In the future, we will try to incorporate the effects of delay, genetics, and treatment for COIVD-19 and diabetes.

\section{Data availability}

The data set we have used are just parameters and initial conditions which we are mentioned in numerical simulation. For data fitting, we have used the data available in [1]. There is no special dataset other than that we have used.

\section{Author's contributions}

S. Anusha : Conceptualization of the idea, Development of the model framework, Analysis and simulation, Writing.

S. Athithan: Development of the model framework, Supervision, Validation and Submission process.

\section{Funding}

There is no funding available for this article. 


\section{Declarations}

Conflict of interest The authors declare that they have no conflict of interest.

\section{References}

1. World Health Organisation. Global Situation. Available at https://covid19.who. int/ Accessed on 30 Jul 2021.

2. Qun Li, Xuhua Guan, Peng Wu, Xiaoye Wang, Lei Zhou, Yeqing Tong, Ruiqi Ren, Kathy SM Leung, Eric HY Lau, Jessica Y Wong, et al. Early transmission dynamics in wuhan, china, of novel coronavirus-infected pneumonia. New England journal of medicine, 2020.

3. Jasper Fuk-Woo Chan, Shuofeng Yuan, Kin-Hang Kok, Kelvin Kai-Wang To, Hin Chu, Jin Yang, Fanfan Xing, Jieling Liu, Cyril Chik-Yan Yip, Rosana Wing-Shan Poon, et al. A familial cluster of pneumonia associated with the 2019 novel coronavirus indicating person-to-person transmission: a study of a family cluster. The lancet, 395(10223):514-523, 2020.

4. Nanshan Chen, Min Zhou, Xuan Dong, Jieming Qu, Fengyun Gong, Yang Han, Yang Qiu, Jingli Wang, Ying Liu, Yuan Wei, et al. Epidemiological and clinical characteristics of 99 cases of 2019 novel coronavirus pneumonia in wuhan, china: a descriptive study. The lancet, 395(10223):507-513, 2020.

5. International Diabetes Federation. Available at https://www.idf.org/aboutdiabetes/ what-is-diabetes/covid-19-and-diabetes/1-covid-19-and-diabetes. html Accessed on 30 Jul 2021.

6. COVID-19 Public Health Emergency of International Concern. Available at https://www.who.int/publications/m/item/ covid-19-public-health-emergency-of-international-concern-(pheic) - global-research-and-innovation-forum. Accessed on 30 Jul 2021.

7. WHO Director-General's opening remarks on COVID-19-11 March $2020 . \quad$ Available at https://www.who.int/director-general/speeches/detail/ who-director-general-s-opening-remarks-at-the-media-briefing-on-covid-19---11-march-2020 Accessed on 30 Jul 2021.

8. World Health Organisation. United States of America Situation. Available at https:// covid19. who. int/region/amro/country/us. Accessed on 30 Jul 2021.

9. World Health Organisation. India Situation. Available at https://covid19.who.int/ region/searo/country/in. Accessed on 30 Jul 2021.

10. World Health Organisation. Brazil Situation. Available at https://covid19.who.int/ region/amro/country/br. Accessed on 30 Jul 2021.

11. World Health Organisation. France Situation. Available at https://covid19.who.int/ region/euro/country/fr. Accessed on 30 Jul 2021.

12. World Health Organisation. Russian Federation Situation. Available at https: / / covid19. who. int/region/euro/country/ru Accessed on 30 Jul 2021.

13. World Health Organisation. Turkey Situation. Available at https://covid19.who.int/ region/euro/country/tr. Accessed on 30 Jul 2021.

14. Chaolin Huang, Yeming Wang, Xingwang Li, Lili Ren, Jianping Zhao, Yi Hu, Li Zhang, Guohui Fan, Jiuyang Xu, Xiaoying Gu, et al. Clinical features of patients infected with 2019 novel coronavirus in wuhan, china. The lancet, 395(10223):497-506, 2020.

15. Kristian Bjorgul, Wendy M Novicoff, and Khaled J Saleh. Evaluating comorbidities in total hip and knee arthroplasty: available instruments. Journal of orthopaedics and traumatology, 11(4):203-209, 2010.

16. Dawei Wang, Bo Hu, Chang Hu, Fangfang Zhu, Xing Liu, Jing Zhang, Binbin Wang, Hui Xiang, Zhenshun Cheng, Yong Xiong, et al. Clinical characteristics of 138 hospitalized patients with 2019 novel coronavirus-infected pneumonia in wuhan, china. Jama, 323(11):1061-1069, 2020.

17. Jing Yang, Ya Zheng, Xi Gou, Ke Pu, Zhaofeng Chen, Qinghong Guo, Rui Ji, Haojia Wang, Yuping Wang, and Yongning Zhou. Prevalence of comorbidities and its effects in patients infected with sarscov-2: a systematic review and meta-analysis. International Journal of Infectious Diseases, 94:91-95, 2020 . 
18. Qiao Shi, Xiaoyi Zhang, Fang Jiang, Xuanzhe Zhang, Ning Hu, Chibu Bimu, Jiarui Feng, Su Yan, Yongjun Guan, Dongxue Xu, et al. Clinical characteristics and risk factors for mortality of covid-19 patients with diabetes in wuhan, china: a two-center, retrospective study. Diabetes care, 43(7):13821391, 2020.

19. Weina Guo, Mingyue Li, Yalan Dong, Haifeng Zhou, Zili Zhang, Chunxia Tian, Renjie Qin, Haijun Wang, Yin Shen, Keye Du, et al. Diabetes is a risk factor for the progression and prognosis of covid19. Diabetes/metabolism research and reviews, 36(7):e3319, 2020.

20. Ernesto Maddaloni and Raffaella Buzzetti. Covid-19 and diabetes mellitus: unveiling the interaction of two pandemics. Diabetes/metabolism research and reviews, 36(7):e33213321, 2020.

21. D Atlas. International diabetes federation. IDF Diabetes Atlas, 8th edn. Brussels, Belgium: International Diabetes Federation, 2017.

22. D Atlas. International diabetes federation. IDF Diabetes Atlas, 9th edn. Brussels, Belgium: International Diabetes Federation, 2019.

23. Gojka Roglic. Who global report on diabetes: A summary. International Journal of Noncommunicable Diseases, 1(1):3, 2016

24. Centres for Disease Control and Prevention (CDC). Coronavirus Disease 2019 (COVID-19). Available at https://www.cdc.gov/coronavirus/2019-ncov/index.html Accessed on $30 \mathrm{Jul} 2021$.

25. Andrea Remuzzi and Giuseppe Remuzzi. Covid-19 and italy: what next? The lancet 395(10231):1225-1228, 2020.

26. Fei Zhou, Ting Yu, Ronghui Du, Guohui Fan, Ying Liu, Zhibo Liu, Jie Xiang, Yeming Wang, Bin Song, Xiaoying $\mathrm{Gu}$, et al. Clinical course and risk factors for mortality of adult inpatients with covid19 in wuhan, china: a retrospective cohort study. The lancet, 395(10229):1054-1062, 2020.

27. Tao Chen, DI Wu, Huilong Chen, Weiming Yan, Danlei Yang, Guang Chen, Ke Ma, Dong Xu, Haijing Yu, Hongwu Wang, et al. Clinical characteristics of 113 deceased patients with coronavirus disease 2019: retrospective study. $b m j, 368,2020$.

28. Wei-jie Guan, Wen-hua Liang, Yi Zhao, Heng-rui Liang, Zi-sheng Chen, Yi-min Li, Xiao-qing Liu, Ru-chong Chen, Chun-li Tang, Tao Wang, et al. Comorbidity and its impact on 1590 patients with covid-19 in china: a nationwide analysis. European Respiratory Journal, 55(5), 2020.

29. Ashu Rastogi, Himika Dogra, and Edward B Jude. Covid-19 and peripheral arterial complications in people with diabetes and hypertension: A systematic review. Diabetes \& Metabolic Syndrome: Clinical Research \& Reviews, page 102204, 2021.

30. Rimesh Pal and Sanjay K Bhadada. Covid-19 and diabetes mellitus: An unholy interaction of two pandemics. Diabetes \& Metabolic Syndrome: Clinical Research \& Reviews, 14(4):513-517, 2020.

31. Md Safaet Hossain Sujan, Rafia Tasnim, Md Saiful Islam, Most Zannatul Ferdous, Md Abdur Rahman Apu, Md Miftah Musfique, and Shahina Pardhan. Covid-19-specific diabetes worries amongst diabetic patients: The role of social support and other co-variates. Primary Care Diabetes, 2021.

32. Antonia Anna Lukito, Raymond Pranata, Joshua Henrina, Michael Anthonius Lim, Sherly Lawrensia, and Ketut Suastika. The effect of metformin consumption on mortality in hospitalized covid-19 patients: a systematic review and meta-analysis. Diabetes \& Metabolic Syndrome: Clinical Research \& Reviews, 2020.

33. M Higazy. Novel fractional order sidarthe mathematical model of covid-19 pandemic. Chaos, Solitons \& Fractals, 138:110007, 2020

34. Piu Samui, Jayanta Mondal, and Subhas Khajanchi. A mathematical model for covid-19 transmission dynamics with a case study of india. Chaos, Solitons \& Fractals, 140:110173, 2020.

35. Olumuyiwa James Peter, Sania Qureshi, Abdullahi Yusuf, Mohammed Al-Shomrani, and Abioye Abioye Idowu. A new mathematical model of covid-19 using real data from pakistan. Results in Physics, 24:104098, 2021.

36. Subhas Khajanchi, Kankan Sarkar, Jayanta Mondal, Kottakkaran Sooppy Nisar, and Sayed F Abdelwahab. Mathematical modeling of the covid-19 pandemic with intervention strategies. Results in Physics, 25:104285, 2021.

37. Prashant Pandey, Yu-Ming Chu, JF Gómez-Aguilar, Hadi Jahanshahi, and Ayman A Aly. A novel fractional mathematical model of covid-19 epidemic considering quarantine and latent time. Results in Physics, 26:104286, 2021.

38. Saddam Mollah and Santosh Biswas. Effect of awareness program on diabetes mellitus: deterministic and stochastic approach. Journal of Applied Mathematics and Computing, pages 1-26, 2020.

39. Manju Agarwal and Rachana Pathak. The impact of awareness programs by media on the spreading and control of non-communicable diseases. International Journal of Engineering, Science and Technology, 6(5):78-87, 2014. 
40. A Boutayeb, EH Twizell, K Achouayb, and A Chetouani. A mathematical model for the burden of diabetes and its complications. Biomedical engineering online, 3(1):1-8, 2004.

41. Abdesslam Boutayeb and Abdelaziz Chetouani. Dynamics of a disabled population in morocco. BioMedical Engineering Online, 2(1):1-6, 2003.

42. M Derouich, A Boutayeb, and EH Twizell. A model of dengue fever. BioMedical Engineering OnLine, 2(1):1-10, 2003.

43. Abdelfatah Kouidere, Lahcen EL Youssoufi, Hanane Ferjouchia, Omar Balatif, and Mostafa Rachik. Optimal control of mathematical modeling of the spread of the covid-19 pandemic with highlighting the negative impact of quarantine on diabetics people with cost-effectiveness. Chaos, Solitons \& Fractals, 145:110777, 2021

44. M Rajalakshmi and Mini Ghosh. Modeling treatment of cancer using virotherapy with generalized logistic growth of tumor cells. Stochastic Analysis and Applications, 36(6):1068-1086, 2018.

45. Akhil Kumar Srivastav, Mini Ghosh, and Peeyush Chandra. Modeling dynamics of the spread of crime in a society. Stochastic Analysis and Applications, 37(6):991-1011, 2019.

46. Kwang Su Kim, Sangil Kim, and Il Hyo Jung. Dynamics of tumor virotherapy: A deterministic and stochastic model approach. Stochastic Analysis and Applications, 34(3):483-495, 2016.

47. Edward J Allen, Linda JS Allen, Armando Arciniega, and Priscilla E Greenwood. Construction of equivalent stochastic differential equation models. Stochastic analysis and applications, 26(2):274 297, 2008.

48. B Mukhopadhyay and R Bhattacharyya. A nonlinear mathematical model of virus-tumor-immune system interaction: deterministic and stochastic analysis. Stochastic Analysis and Applications, 27(2):409-429, 2009.

49. David L Smith and F Ellis McKenzie. Statics and dynamics of malaria infection in anopheles mosquitoes. Malaria journal, 3(1):1-14, 2004.

50. Klaus Dietz. The estimation of the basic reproduction number for infectious diseases. Statistical methods in medical research, 2(1):23-41, 1993.

51. L Arriola and J Hyman. Lecture notes, forward and adjoint sensitivity analysis: with applications in dynamical systems. Linear Algebra and Optimisation Mathematical and Theoretical Biology Institute, Summer, 2005.

52. Nakul Chitnis, James M Hyman, and Jim M Cushing. Determining important parameters in the spread of malaria through the sensitivity analysis of a mathematical model. Bulletin of mathematical biology, 70(5):1272, 2008.

53. Matthew C Riddle, John B Buse, Paul W Franks, William C Knowler, Robert E Ratner, Elizabeth Selvin, Deborah J Wexler, and Steven E Kahn. Covid-19 in people with diabetes: urgently needed lessons from early reports. Diabetes Care, 43(7):1378-1381, 2020.

54. Emma Barron, Chirag Bakhai, Partha Kar, Andy Weaver, Dominique Bradley, Hassan Ismail, Peter Knighton, Naomi Holman, Kamlesh Khunti, Naveed Sattar, et al. Associations of type 1 and type 2 diabetes with covid-19-related mortality in england: a whole-population study. The lancet Diabetes \& endocrinology, 8(10):813-822, 2020.

55. Feng Gao, Kenneth I Zheng, Xiao-Bo Wang, Qing-Feng Sun, Ke-Hua Pan, Ting-Yao Wang, YongPing Chen, Giovanni Targher, Christopher D Byrne, Jacob George, et al. Obesity is a risk factor for greater covid-19 severity. Diabetes care, 43(7):e72-e74, 2020.

56. Matt Arentz, Eric Yim, Lindy Klaff, Sharukh Lokhandwala, Francis X Riedo, Maria Chong, and Melissa Lee. Characteristics and outcomes of 21 critically ill patients with covid-19 in washington state. Jama, 323(16):1612-1614, 2020.

57. Ilhan Satman, Ibrahim Demirci, Cem Haymana, Ilker Tasci, Serpil Salman, Naim Ata, Selcuk Dagdelen, Ibrahim Sahin, Rifat Emral, Erman Cakal, et al. Unexpectedly lower mortality rates in covid19 patients with and without type 2 diabetes in istanbul. Diabetes Research and Clinical Practice, 174:108753, 2021

58. Yuchen Chen, Dong Yang, Biao Cheng, Jian Chen, Anlin Peng, Chen Yang, Chong Liu, Mingrui Xiong, Aiping Deng, Yu Zhang, et al. Clinical characteristics and outcomes of patients with diabetes and covid-19 in association with glucose-lowering medication. Diabetes care, 43(7):1399-1407, 2020.

59. Yuan Yuan and Linda JS Allen. Stochastic models for virus and immune system dynamics. Mathematical biosciences, 234(2):84-94, 2011. 